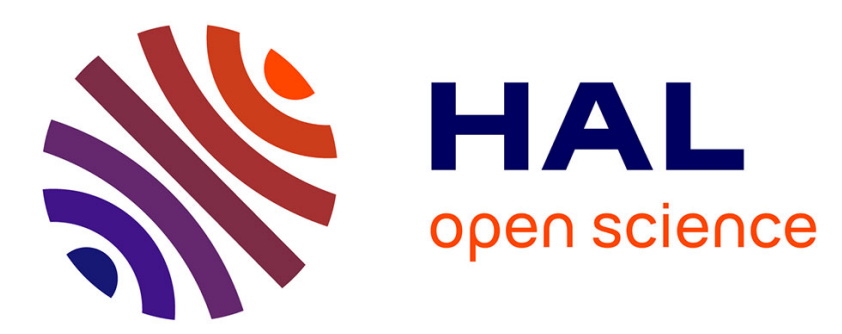

\title{
On the identification of multiple space dependent ionic parameters in cardiac electrophysiology modelling
}

Yassine Abidi, Mourad Bellassoued, Moncef Mahjoub, Nejib Zemzemi

\section{To cite this version:}

Yassine Abidi, Mourad Bellassoued, Moncef Mahjoub, Nejib Zemzemi. On the identification of multiple space dependent ionic parameters in cardiac electrophysiology modelling. [Research Report] INRIA. 2017. hal-01567714

\section{HAL Id: hal-01567714 \\ https://hal.inria.fr/hal-01567714}

Submitted on 24 Jul 2017

HAL is a multi-disciplinary open access archive for the deposit and dissemination of scientific research documents, whether they are published or not. The documents may come from teaching and research institutions in France or abroad, or from public or private research centers.
L'archive ouverte pluridisciplinaire HAL, est destinée au dépôt et à la diffusion de documents scientifiques de niveau recherche, publiés ou non, émanant des établissements d'enseignement et de recherche français ou étrangers, des laboratoires publics ou privés. 


\title{
ON THE IDENTIFICATION OF MULTIPLE SPACE DEPENDENT IONIC PARAMETERS IN CARDIAC ELECTROPHYSIOLOGY MODELLING
}

\author{
YASSINE ABIDI ${ }^{1}$, MOURAD BELLASSOUED $^{1}$, MONCEF MAHJOUB $^{1}$ AND NEJIB ZEMZEMI $^{2}$
}

\begin{abstract}
In this paper, we consider the inverse problem of space dependent multiple ionic parameters identification in cardiac electrophysiology modelling from a set of observations. We use the monodomain system known as a state-of-the-art model in cardiac electrophysiology and we consider a general Hodgkin-Huxley formalism to describe the ionic exchanges at the microscopic level. This formalism covers many physiological transmembrane potential models including those in cardiac electrophysiology. Our main result is the proof of the uniqueness and a Lipschitz stability estimate of ion channels conductance parameters based on some observations on an arbitrary subdomain. The key idea is a Carleman estimate for a parabolic operator with multiple coefficients and an ordinary differential equation system.

Keywords: Lipschitz stability estimate, Carleman estimate, cardiac electrophysiology modelling, monodomain system, Hodgkin-Huxley model, ionic parameters identification.
\end{abstract}

\section{INTRODUCTION}

The electric wave propagation in the heart can be represented by a non-linear reaction-diffusion system coupled to an ordinary differential equation system called the bidomain model [39, 15]. It takes into account the electrical potential both in the intra-cellular and extra-cellular domains. The coupled system describes the evolution of the transmembrane and the extracellular potentials in the heart. This mathematical model can be formulated as a three-field system (ionic state, transmembrane and extracellular potentials) coupling a non-linear reaction-diffusion equation to an elliptic equation and a non-linear system of ODEs. A simplification of this model is given by the so-called monodomain system, it consists of one parabolic non linear PDE coupled to an ODE system. This model is equivalent to the bidomain model when ratios of the intracellular conductivity anisotropy are close to those in the extracellular domains. It is widely used in the computational electrophysiology community because it is computationally much cheaper than the full bidomain model.

Recent works in the computational cardiology community have been dedicated to the personalization of mathematical models [32, 37, 11, 6, 10, 8, 40] using different approaches. The idea is to assimilate different measured data and automatically calibrate the parameters of the computational model in order to make it behave like the measured observations. These approaches are very attractive because, in the future, they could potentially help in improving the diagnosis of the heart condition and in planning therapeutic interventions. On the other hand, very few works have been interested in studying the stability of the parameters identification inverse problems for such models. To the best of our knowledge, only two works have been performed on this subject [36, 5]. Both of works still have some limitations that will be addressed in this paper. The first limitation is that they only treat simplified ionic models: The first paper [36] shows the stability of the identification a reaction parameter in the Mitchell-Schaeffer model [38]. The second work [5] treats the stability of a reaction parameter in the FitzHugh-Nagumo model [14] and a parameter in the ODE system of the model but separately. The second limitation is they do not treat the stability of identifying a set of parameters at the same time. The main novelties in this paper with respect to the cited works are twofold: First, we present a methodology for physiologically-detailed ionic models covering models under a general

Date: June 28, 2017. 
Hodgkin-Huxley formalism [19] (HH) including the Beeler-Reuter model [2] and the Luo-Rudy I model [34] (LRI) which are one of the most popular ionic models in the cardiac electrophysiology modelling community and many other transmembrane potential models. Second, we present an approach and some conditions under which one could prove the stability of multiple parameters identification problem. Here, we are concerned about ion channels conductance parameters.

The paper is organized as follows: In the next section, we briefly recall the general structure of cardiac cellular membrane models describing the transmembrane potential and the ionic exchange at the cell membrane. Then, we present the monodomain model describing the electrical wave propagation and recall some existence, uniqueness and regularity results that have been shown in [42]. We also establish new regularity results that would help us in the stability analysis. In section $\$ 3$, we announce the main stability result including the conditions we need for the identification of multiple parameters. The proof of the main result is divided into two sections. In section $\$ 4$, we prove the global Carleman inequality for the reaction-diffusion system. Most of the non-classical parts of the proof of the main result are presented in section \$5 where we prove the stability estimate of conductances parameters.

\section{MATHEMATICAL MODELS FOR THE ELECTRICAL WAVE PROPAGATION}

In this section, we first present the general structure of physiologically-detailed cardiac cellular membrane models that we will use in this paper. Then, we introduce the monodomain model coupling a reaction diffusion parabolic equation to a physiological ODE system.

2.1. General structure of cardiac cellular membrane models. The ionic current throughout channels of the membrane is modulated by the transmembrane potential $v:=u_{i}-u_{e}$, where $u_{i}$ and $u_{e}$ are respectively the intra- and extra-cellular potentials, the gating variables $\mathbf{w}:=\left(w_{1}, \ldots, w_{k}\right)$ and by the ionic intracellular concentration variables $\mathbf{z}:=\left(z_{1}, \ldots, z_{m}\right)$. In the membrane model, the ionic current $I_{i o n}$ has the following general structure [16]:

$$
I_{\text {ion }}(\bar{\varrho}, v, \mathbf{w}, \mathbf{z})=\sum_{i=1}^{N} \bar{\varrho}_{i} y_{i}(v) \prod_{j=1}^{k} w_{j}^{p_{j, i}}\left(v-E_{i}(\mathbf{z})\right),
$$

where $N$ is the number of ionic currents, $\varrho_{i}$ is the maximal conductance associated with the $i^{t h}$ current, $y_{i}$ is a gating function depending only on the membrane potentiel $v, p_{j, i}$ are positive integers exponents and $E_{i}$ is the reversal potential for the $i^{t h}$ current $I_{i}$, which is the related equilibrium (Nernst) potential and is given by

$$
E_{i}(\mathbf{z})=\bar{\gamma}_{i} \log \left(\frac{z_{e}}{z_{i}}\right), \quad \mathbf{z}=\left(z_{1}, \ldots, z_{m}\right)
$$

where $\bar{\gamma}_{i}$ is a constant and $z_{i}, i=1, \ldots, m$, are the intracellular concentrations. The constant $z_{e}$ denotes an extracellular concentration. Here, we use the regularized form of the variable $y_{i}(v)$ in hyperbolic functions such as $s h, c h, t h$ introduced in [13]. In this case, $y_{i}(v)$ is a $\mathcal{C}^{\infty}$ function with respect to the variable $v$ for $i=1 \ldots N$.

The dynamics of the gating variable $\mathbf{w}$ is described in the Hodgkin-Huxley formalism by a system of ordinary differential equations which when $w_{j}$ is a gating variable $\left(0 \leqslant w_{j} \leqslant 1\right)$ are governed by the following equation,

$$
\partial_{t} w_{j}=F_{j}\left(v, w_{j}\right):=\alpha_{j}(v)\left(1-w_{j}\right)-\beta_{j}(v) w_{j}, \quad j=1, \ldots, k,
$$


where $\alpha_{j}$ and $\beta_{j}$ are positive rational functions of exponentials in $v$. A general expression for both $\alpha_{j}$ and $\beta_{j}$ is given by

$$
\frac{\mu_{1} e^{\mu_{2}\left(v-v_{n}\right)}+\mu_{3}\left(v-v_{n}\right)}{1+\mu_{4} e^{\mu_{5}\left(v-v_{n}\right)}},
$$

where $\mu_{1}, \mu_{3}, \mu_{4}, v_{n}$ are non-negative constants and $\mu_{2}, \mu_{5}$ are positive constants.

The dynamics of the ionic concentration variables $\mathbf{z}$ is described by the additional system of ordinary differential equations:

$$
\partial_{t} z_{i}=G_{i}(\bar{\varrho}, v, \mathbf{w}, \mathbf{z}):=-J_{i}\left(\bar{\varrho}, v, \mathbf{w}, \log z_{i}\right)+H_{i}(\bar{\varrho}, v, \mathbf{w}, \mathbf{z}), \quad i=1, \ldots, m,
$$

where

$$
J_{i} \in \mathcal{C}^{2}\left(\mathbb{R}_{+}^{*} \times \mathbb{R} \times \mathbb{R}^{k} \times \mathbb{R}\right), \quad 0<g_{*}(\mathbf{w}) \leqslant \frac{\partial J_{i}}{\partial \tau}(\bar{\varrho}, v, \mathbf{w}, \tau) \leqslant g^{*}(\mathbf{w}), \quad\left|\frac{\partial J_{i}}{\partial v}(\bar{\varrho}, v, \mathbf{w}, 0)\right| \leqslant L_{v}(\mathbf{w}),
$$

$g_{*}, g^{*}, L_{v}$ belong to $\mathcal{C}^{1}\left(\mathbb{R}^{k}, \mathbb{R}_{+}\right)$, and

$$
H_{i} \in \mathcal{C}^{2}\left(\mathbb{R}_{+}^{*} \times \mathbb{R} \times \mathbb{R}^{k} \times(0,+\infty)^{m}\right) \cap \operatorname{Lip}\left(\mathbb{R}_{+}^{*} \times \mathbb{R} \times[0,1]^{k} \times(0,+\infty)^{m}\right) .
$$

One could find in the literature many refined models based on Hodgkin-Huxley formalism taking into account different quantities. For example, we recall here the following models: Beeler-Reuter ([2], $N=$ $4, k=6, m=1$ ), phase-I Luo-Rudy ([34], $N=6, k=6, m=1)$, phase-II Luo-Rudy ([35], $N=10, k=$ $6, m=5)$.

2.2. Monodomain system with generalized ionic models. In this paper, we consider the monodomain system. It describes the propagation of the electric wave in the heart and is given by

$$
\begin{cases}\partial_{t} v-\operatorname{div}(\boldsymbol{\sigma} \nabla v)=I_{a p p}+I_{i o n}(\bar{\varrho}, v, \mathbf{w}, \mathbf{z}) & \text { in } Q \equiv \Omega \times(0, T), \\ \partial_{t} \mathbf{w}=\boldsymbol{F}(v, \mathbf{w}) & \text { in } Q, \\ \partial_{t} \mathbf{z}=\boldsymbol{G}(\bar{\varrho}, v, \mathbf{w}, \mathbf{z}) & \text { in } Q, \\ \sigma \nabla v \cdot \nu=0 & \text { on } \Sigma \equiv \partial \Omega \times(0, T) .\end{cases}
$$

Here $\Omega \subset \mathbb{R}^{3}$ is a bounded domain representing the cardiac tissue whose boundary $\partial \Omega$. The time domain is given by $[0, T]$. We also denote by $Q_{t}:=\Omega \times(0, t)$, for any time $t>0$. The variable $v$, denotes the action potential and $\boldsymbol{\sigma}:=\boldsymbol{\sigma}_{i}\left(\boldsymbol{\sigma}_{i}+\boldsymbol{\sigma}_{e}\right)^{-1} \boldsymbol{\sigma}_{e}$ is the bulk conductivity where $\boldsymbol{\sigma}_{i}$ and $\boldsymbol{\sigma}_{e}$ are the intra- and extracellular conductivity tensors and $\boldsymbol{\nu}=\boldsymbol{\nu}(x)=\left(\nu_{1}(x), \nu_{2}(x), \nu_{3}(x)\right)$ is the external unit normal vector to $\partial \Omega$ at $x$. The term $I_{a p p}$ is an applied electrical current. We will consider that it satisfies the following regularity.

$$
I_{a p p} \in L^{p}\left(0, T ; L^{2}(\Omega)\right) \cap H^{1}\left(0, T ; H^{2}(\Omega)\right), \quad p>4 .
$$

The ionic current $I_{i o n}$ and the functions $\boldsymbol{F}$ and $\boldsymbol{G}$ depends of the considered ionic model. We assume that the conductivities of the intracellular and extracellular $\boldsymbol{\sigma}_{i}, \boldsymbol{\sigma}_{e} \in\left[\mathcal{C}^{1}(\bar{\Omega})\right]^{3 \times 3}$ are symmetric and uniformly positive definite, i.e, there exist $\alpha_{i}>0$ and $\alpha_{e}>0$ such that,

$$
\xi^{\top} \boldsymbol{\sigma}_{i}(x) \xi \geqslant \alpha_{i}|\xi|^{2}, \quad \xi^{\top} \boldsymbol{\sigma}_{e}(x) \xi \geqslant \alpha_{e}|\xi|^{2}, \quad \forall \xi \in \mathbb{R}^{3},
$$

and that the coefficients $\boldsymbol{\sigma}_{j k}, j, k=1,2,3$ of the matrix $\boldsymbol{\sigma}$, satisfy the uniform ellipticity: there exists a constant $\mu>0$ such that

$$
\mu|\xi|^{2} \leqslant \xi^{\top} \boldsymbol{\sigma} \xi, \quad \forall \xi \in \mathbb{R}^{3}
$$


We set

$$
|\nabla u|_{\boldsymbol{\sigma}}^{2}:=\boldsymbol{\sigma} \nabla u \cdot \nabla u=\sum_{j, k=1}^{3} \boldsymbol{\sigma}_{j k} \partial_{j} u \partial_{k} u .
$$

To system (2.8), we attach initial conditions :

$$
\begin{aligned}
& v(\cdot, 0)=v_{0} \in H^{2}(\Omega), \\
& \mathbf{w}(\cdot, 0)=\mathbf{w}_{0}, \quad \mathbf{w}_{0}: \Omega \rightarrow[0,1]^{k}, \text { measurable, } \\
& \mathbf{z}(\cdot, 0)=\mathbf{z}_{0} \in L^{2}(\Omega)^{m}, \text { with } \log \mathbf{z}_{0}:=\left(\log z_{0,1}, \ldots, \log z_{0, m}\right) \in L^{2}(\Omega)^{m} .
\end{aligned}
$$

Before stating the main results, we recall the following lemma on the unique existence of a strong solution to problem (2.8). The proof is provided in [42] and is based on a fixed point method.

Lemma 2.1. Assume that $\Omega$ is $\mathcal{C}^{1,1}$ and $\left(v_{0}, \mathbf{z}_{0}, \mathbf{w}_{0}\right)$ satisfying (2.12). Let us take as given the ionic currents satisfying (2.1)-(2.2), the dynamics of the gating variables $\boldsymbol{F}(v, \mathbf{w})$ satisfying (2.3), the dynamics of the ionic concentration $\boldsymbol{G}(\bar{\varrho}, v, \mathbf{w}, \mathbf{z})$, satisfying (2.5)-(2.7). Then, there exists a unique solution $(v, \mathbf{w}, \mathbf{z})$ of (2.8) with initial condition (2.14) with the regularity

$$
\begin{aligned}
v:=u_{i}-u_{e} \in W^{1, p}\left(0, T ; L^{2}(\Omega)\right) \cap L^{2}\left(0, T ; H^{2}(\Omega)\right) \cap \mathcal{C}^{0}\left([0, T], \mathcal{C}^{0}(\Omega)\right), \quad \text { for } p>4, \\
\mathbf{w}: Q \rightarrow[0,1]^{k} \quad \text { measurable, } \mathbf{z}: Q \rightarrow(0,+\infty)^{m} \quad \text { measurable, } \\
w_{j}(x, .) \in \mathcal{C}^{1}(0, T) \cap \mathcal{C}^{0}([0, T]), \quad \text { for } \text { a.e. }, \quad x \in \Omega, \quad j=1, \ldots, k, \\
z_{i}(x, .) \in \mathcal{C}^{1}(0, T) \cap \mathcal{C}^{0}([0, T]), \quad \text { for } \text { a.e., } \quad x \in \Omega, \quad i=1, \ldots, m, \\
\mathbf{z} \in H^{1}\left(0, T ; L^{2}(\Omega)\right)^{m} \cap L^{\infty}(Q)^{m}, \quad \log \mathbf{z}:=\left(\log z_{1}, \ldots, \log z_{m}\right) \in L^{\infty}(Q)^{m} .
\end{aligned}
$$

Moreover there exists a constant $C>0$, independant of $v, \mathbf{w}, \mathbf{z}$, such that

$$
|\mathbf{z}(x, t)| \leqslant C\left(1+\left|\mathbf{z}_{0}(x)\right|+\|v(x)\|_{L^{2}(0, t)}\right), \quad \text { a.e., } x \in \Omega,
$$

and

$$
|\log \mathbf{z}(x, t)|+\left|\partial_{t} \mathbf{z}(x, t)\right| \leqslant C\left(1+\left|\mathbf{z}_{0}(x)\right|+\|v(x)\|_{\mathcal{C}^{0}(0, t)}\right), \quad \text { a.e., } x \in \Omega,
$$

$\forall t \in[0, T]$, for a.e. $x \in \Omega$.

Also, there exists $M_{\infty}>0$, depending on the data of the problem, such that:

$$
\sup \{|v(x, t)|:(x, t) \in Q\} \leqslant M_{\infty} .
$$

Remark 2.1. This lemma has been proved in [42] for constant ionic model parameters. The same result is preserved when considering that these parameters are in $\mathcal{C}^{0}(\bar{\Omega})$.

Since we are interested in identifying ion channels conductance parameters, from now on, we will suppose that all the parameters but $\bar{\varrho}$ are constant. We will consider that conductance parameters $\bar{\varrho} \in\left(H^{3}(\Omega)\right)^{N}$ which already, using Sobolev's embeddings, give us the $\mathcal{C}^{0}(\bar{\Omega})$ regularity.

In the following lemma, we prove a lower bound on $\mathbf{z}$ when 2.5$)-(2.7)$ holds. The proof is based on some results in [41].

Lemma 2.2. Under the same assumptions as in Lemma 2.1. there exists a constant $C>0$, depending on $m, T$ such that, $\forall i=1, \ldots, m$,

$$
z_{i}(x, t) \geqslant \exp \left[-C\left(1+\left\|\mathbf{z}_{0}\right\|_{L^{\infty}}+\|v\|_{\mathcal{C}^{0}\left([0, T], \mathcal{C}^{0}(\Omega)\right)}\right)\right]>0, \quad \forall t \in[0, T], \text { a.e., } x \in \Omega .
$$


Proof. Since we want to show 2.16 and $\exp \left[-C\left(1+\left\|\mathbf{z}_{0}\right\|_{L^{\infty}}+\|v\|_{\mathcal{C}^{0}\left([0, T], \mathcal{C}^{0}(\Omega)\right)}\right)\right]<1$, we can limit the study to $z_{i}<1$. We consider the equation

$$
\partial_{t} z_{i}=-J_{i}\left(\bar{\varrho}, v, \mathbf{w}, \log z_{i}\right)+H_{i}(\bar{\varrho}, v, \mathbf{w}, \mathbf{z}) .
$$

We note that, for $i=1, \ldots, m$, we can write

$$
J_{i}\left(\bar{\varrho}, v, \mathbf{w}, \log z_{i}\right)=J_{i}(\bar{\varrho}, v, \mathbf{w}, 0)+\frac{J_{i}\left(\bar{\varrho}, v, \mathbf{w}, \log z_{i}\right)-J_{i}(\bar{\varrho}, v, \mathbf{w}, 0)}{\log z_{i}} \log \left(z_{i}\right) .
$$

Owing to 2.6 , there exists a constant $\bar{L}>0$, depending on $L_{v}$, and a constants $\underline{G}, \bar{G}>0$ such that

$$
J_{i}(\bar{\varrho}, v, \mathbf{w}, 0) \leqslant \bar{L}(1+|v|), \quad \forall(v, \mathbf{w}) \in \mathbb{R} \times[0,1]^{k},
$$

and

$$
\underline{G} \leqslant \frac{J_{i}\left(\bar{\varrho}, v, \mathbf{w}, \log z_{i}\right)-J_{i}(\bar{\varrho}, v, \mathbf{w}, 0)}{\log z_{i}} \leqslant \bar{G}, \quad \forall\left(v, \mathbf{w}, z_{i}\right) \in \mathbb{R} \times[0,1]^{k} \times(0,+\infty) .
$$

Moreover, by hypothesis 2.7] there exists a constant $\Lambda>0$ such that

$$
\left|H_{i}(\bar{\varrho}, v, \mathbf{w}, \mathbf{z})\right| \leqslant \Lambda(1+|v|+|\mathbf{z}|), \quad \forall(v, \mathbf{w}, \mathbf{z}) \in \mathbb{R} \times[0,1]^{k} \times(0,+\infty)^{m} .
$$

Using (2.17)-2.19), we find

$$
\partial_{t} z_{i}(x, t) \geqslant-\bar{L}(1+|v|)-\underline{G} \log z_{i}-\Lambda(1+|v|+|\mathbf{z}|) .
$$

Then, from (2.13), if

$$
\underline{G} \log z_{i} \leqslant-\bar{L}\left(1+\|v(x)\|_{C^{0}(0, t)}\right)-\Lambda\left(1+\|v(x)\|_{C^{0}(0, t)}+C\left(1+\left|\mathbf{z}_{0}\right|+\|v(x)\|_{L^{2}(0, t)}\right)\right),
$$

then

$$
\partial_{t} z_{i}(x, t) \geqslant 0 \text {. }
$$

Since

$$
\|v(x)\|_{L^{2}(0, t)} \leqslant \sqrt{T}\|v(x)\|_{C^{0}(0, t)}
$$

we deduce from 2.14 that

$$
z_{i}(x, t) \geqslant \exp \left[-C\left(1+\left|\mathbf{z}_{0}\right|_{L^{\infty}}+\|v\|_{\mathcal{C}^{0}(0, t)}\right)\right] .
$$

Let as now consider the case

$$
\underline{G} \log z_{i} \geqslant-\bar{L}\left(1+\|v(x)\|_{C^{0}(0, t)}\right)-\Lambda\left(1+\|v(x)\|_{C^{0}(0, t)}+C\left(1+\left|\mathbf{z}_{0}\right|+\|v(x)\|_{L^{2}(0, t)}\right)\right),
$$

then

$$
z_{i}(x, t) \geqslant \exp \left[-\frac{1}{\underline{G}}\left(\bar{L}\left(1+\|v(x)\|_{C^{0}(0, t)}\right)+\Lambda\left(1+\|v(x)\|_{C^{0}(0, t)}+C\left(1+\left|\mathbf{z}_{0}\right|+\|v(x)\|_{L^{2}(0, t)}\right)\right)\right)\right] .
$$

This completes the proof.

Now, we will establish two propositions dealing with the regularity of the system (2.8) solution. These regularities would be useful in the parameters estimations that will be presented in Section $\$ 5$. The aim is to improve the regularity results given in Lemma 2.1 in order to satisfy the assumptions that would be taken in the stability result. The proofs of the two following Propositions 2.1 and 2.2 are provided in the Appendix. 
Proposition 2.1. Let $(v, \mathbf{w}, \mathbf{z})$ be the solution of equations system (2.8), with initial conditions $v_{0}, \mathbf{w}_{0}$ and $\mathbf{z}_{0}$. If $v_{0} \in H^{2}(\Omega), \mathbf{w}_{0} \in L^{2}(\Omega)^{k}, \mathbf{z}_{0} \in L^{2}(\Omega)^{m}$ and $I_{\text {app }}$ verify the regularity (2.9), then

$$
\begin{gathered}
v \in W^{1, \infty}\left(0, T ; H^{1}(\Omega)\right) \cap L^{\infty}\left(0, T ; H^{2}(\Omega)\right) \cap H^{1}\left(0, T ; H^{1}(\Omega)\right), \\
\mathbf{w} \in W^{1, \infty}\left(0, T ; L^{2}(\Omega)\right)^{k}, \quad \text { and } \quad \mathbf{z} \in W^{1, \infty}\left(0, T ; L^{2}(\Omega)\right)^{m} .
\end{gathered}
$$

Moreover if

$$
\mathbf{w}_{0} \in H^{1}(\Omega)^{k}, \quad \text { and } \quad \mathbf{z}_{0} \in H^{1}(\Omega)^{m}
$$

then

$$
\mathbf{w} \in H^{1}\left(0, T ; H^{1}(\Omega)\right)^{k}, \quad \text { and } \quad \mathbf{z} \in H^{1}\left(0, T ; H^{1}(\Omega)\right)^{m} .
$$

Also, if $v_{0} \in H^{3}(\Omega)$, then

$$
\begin{aligned}
& v \in H^{2}\left(0, T ; L^{2}(\Omega)\right), \\
& \mathbf{w} \in H^{2}\left(0, T ; L^{2}(\Omega)\right)^{k}, \quad \text { and } \quad \mathbf{z} \in H^{2}\left(0, T ; L^{2}(\Omega)\right)^{m} .
\end{aligned}
$$

Proposition 2.2. Let the initial condition of $(2.8)$ be such that $v_{0} \in H^{4}(\Omega), \mathbf{w}_{0} \in H^{2}(\Omega)^{k}, \mathbf{z}_{0} \in H^{2}(\Omega)^{m}$ and $I_{\text {app }}$ verify the regularity (2.9). Then the solution of (2.8) satisfies

$$
\begin{gathered}
v \in H^{1}\left(0, T ; H^{3}(\Omega)\right) \cap W^{1, \infty}\left(0, T ; H^{2}(\Omega)\right) \cap H^{3}\left(0, T ; L^{2}(\Omega)\right), \\
\mathbf{w} \in W^{1, \infty}\left(0, T ; H^{2}(\Omega)\right)^{k} \cap L^{\infty}\left(0, T ; H^{2}(\Omega)\right)^{k}, \\
\mathbf{z} \in W^{1, \infty}\left(0, T ; H^{2}(\Omega)\right)^{m} \cap L^{\infty}\left(0, T ; H^{2}(\Omega)\right)^{m} .
\end{gathered}
$$

Moreover if

$$
\mathbf{w}_{0} \in H^{3}(\Omega)^{k}, \quad \text { and } \quad \mathbf{z}_{0} \in H^{3}(\Omega)^{m}
$$

then

$$
\begin{aligned}
& \mathbf{w} \in W^{1, \infty}\left(0, T ; H^{3}(\Omega)\right)^{k} \hookrightarrow H^{1}\left(0, T ; H^{3}(\Omega)\right)^{k} \\
& \mathbf{z} \in W^{1, \infty}\left(0, T ; H^{3}(\Omega)\right)^{m} \hookrightarrow H^{1}\left(0, T ; H^{3}(\Omega)\right)^{m} .
\end{aligned}
$$

\section{INVERSE PROBLEM: MAIN RESULT}

Let $t_{0} \in(0, T)$ (without loss of generality we can choose $\left.t_{0}=T / 2\right)$ and $\omega \subset \Omega$ be an arbitrary open subset of $\Omega$. Let us also select $I_{a p p}^{\ell}, 1 \leqslant \ell \leqslant N$ suitably in order to determine $\bar{\varrho}(x)=\left(\varrho_{1}(x), \ldots, \bar{\varrho}_{N}(x)\right)$, from the observation data $\left(v_{\ell}, \mathbf{w}_{\ell}, \mathbf{z}_{\ell}\right)_{\mid \omega \times(0, T)}$ and $\left(v_{\ell}\left(x, t_{0}\right), \mathbf{w}_{\ell}\left(x, t_{0}\right), \mathbf{z}_{\ell}\left(x, t_{0}\right)\right), x \in \Omega, 1 \leqslant \ell \leqslant N$.

In the formulation of the inverse problem, the initial values are also unknown. The non-homogeneous terms $I_{a p p}^{\ell}, 1 \leqslant \ell \leqslant N$, re consideerd as input sources to system 2.8 . Then we determine $\bar{\varrho}_{i}(x), x \in \Omega$ by observation data $\left(v_{\ell}, \mathbf{w}_{\ell}, \mathbf{z}_{\ell}\right)_{\mid \omega \times(0, T)}$ and $\left(v_{\ell}\left(x, t_{0}\right), \mathbf{w}_{\ell}\left(x, t_{0}\right), \mathbf{z}_{\ell}\left(x, t_{0}\right)\right), x \in \Omega, 1 \leqslant \ell \leqslant N$, which are refarded as outputs.

We shall determine $\bar{\varrho}_{i}, 1 \leqslant i \leqslant N$ in the neighbourhood of some known set of coefficients $\bar{\varrho}_{i}^{(2)}$. We shall denote by $\bar{\varrho}_{i}$ the unknown set coefficients. Our main concern is the stability estimate for the inverse problem: Estimate $\left\|\varrho^{(1)}-\bar{\varrho}^{(2)}\right\|_{L^{2}(\Omega)^{N}}$ by suitable norms of observation data $\left(v_{\ell}^{(1)}-v_{\ell}^{(2)}, \mathbf{w}_{\ell}^{(1)}-\mathbf{w}_{\ell}^{(2)}, \mathbf{z}_{\ell}^{(1)}-\right.$ $\left.\mathbf{z}_{\ell}^{(2)}\right)_{\mid \omega \times(0, T)}$ and $\left(v_{\ell}^{(1)}-v_{\ell}^{(2)}, \mathbf{w}_{\ell}^{(1)}-\mathbf{w}_{\ell}^{(2)}, \mathbf{z}_{\ell}^{(1)}-\mathbf{z}_{\ell}^{(2)}\right)\left(x, t_{0}\right), x \in \Omega, 1 \leqslant \ell \leqslant N$. The stability estimate is a fundamental mathematical subject in the inverse problem and immediately yields the uniqueness. Stability estimates for inverse problems are not only important from the theoretical viewpoint, but also useful 
for numerical algorithms. In particular, by Cheng and Yamamoto [9] for example, a stability estimate gives convergence rates of Tikhonov regularized solutions, which are widely used as approximating solutions to the inverse problems.

Our inverse problem is related to determination of multiple ionic parameter of a non linear parabolic reaction diffusion system coupled with an ordinary differential equations. To the authors' best knowledge, there are no papers on the determination of multiple coefficients of multiscale mathematical models in cardiac electrophysiology, although we have an available methodology which was initiated by Bukhgeim and Klibanov [7]. The determination of multiple coefficients requires repeated observations, and the application of the method in [45] needs independent consideration.

Moreover, since we aim at the global stability in the whole domain $\Omega$ by means of data on an arbitrary small subset $\omega \subset \Omega$, we have to establish a relevant Carleman estimate (Theorem 3.1 below).

In order to formulate our results, we need to introduce the following notations: For a sequence functions $\left(\widetilde{v}_{\ell}, \widetilde{\mathbf{w}}_{\ell}, \widetilde{\mathbf{z}}_{\ell}\right) \in H^{3}(\Omega) \times \mathcal{C}^{1}(\Omega)^{k} \times \mathcal{C}^{1}(\Omega)^{m}$, we define the $N \times N$ matrix $\Lambda$ as follows

$$
\Lambda\left(\widetilde{v}_{\ell}(x), \widetilde{\mathbf{w}}_{\ell}(x), \widetilde{\mathbf{z}}_{\ell}(x)\right)=\left(\begin{array}{cccc}
S_{1,1}(x) & S_{2,1}(x) & \ldots & S_{N, 1}(x) \\
S_{1,2}(x) & S_{2,2}(x) & \ldots & S_{N, 2}(x) \\
\vdots & \vdots & \ddots & \vdots \\
S_{1, N}(x) & S_{2, N}(x) & \ldots & S_{N, N}(x)
\end{array}\right),
$$

where

$$
S_{i, \ell}(x)=y_{i}\left(\widetilde{v}_{\ell}(x)\right)\left(\widetilde{v}_{\ell}(x)-E_{i}\left(\widetilde{\mathbf{z}}_{\ell}(x)\right)\right) \prod_{j=1}^{k}\left(\widetilde{w}_{\ell}\right)_{j}^{p_{j, i}}(x), 1 \leqslant \ell, i \leqslant N .
$$

Let us fix constant $M_{0}>0$. We introduce an admissible set of unknown coefficients vector $\bar{\varrho}$ by

$$
\mathcal{A}=\left\{\bar{\varrho} \in H^{3}(\Omega)^{N},\|\bar{\varrho}\|_{l^{2}\left(H^{3}(\Omega)^{N}\right)} \leqslant M_{0}\right\} .
$$

We obtain the following stability result.

Theorem 3.1. Let $t_{0} \in(0, T), \omega$ be a subdomain of $\Omega$ and let $\bar{\varrho}^{(2)} \in \mathcal{A}$ be arbitrary fixed. We assume that $I_{\text {app }}^{\ell} \in L^{p}\left(0, T ; L^{2}(\Omega)\right) \cap H^{1}\left(0, T ; H^{2}(\Omega)\right), p>4,1 \leqslant \ell \leqslant N$, satisfy

$$
\operatorname{det}\left(\Lambda\left(v_{\ell}^{(2)}\left(x, t_{0}\right), \mathbf{w}_{\ell}^{(2)}\left(x, t_{0}\right), \mathbf{z}_{\ell}^{(2)}\left(x, t_{0}\right)\right)\right) \neq 0, \quad \forall x \in \Omega .
$$

Here $\left(v_{\ell}^{(2)}, \mathbf{w}_{\ell}^{(2)}, \mathbf{z}_{\ell}^{(2)}\right)$ is the solution of $(2.8)$ with $\bar{\varrho}=\bar{\varrho}^{(2)}$ and $I_{\text {app }}=I_{\text {app }}^{\ell}$. Furthermore, we assume that

$$
\left\|v_{\ell}^{(2)}\right\|_{\mathcal{C}^{0}\left([0, T] ; \mathcal{C}^{1}(\bar{\Omega})\right)}+\left\|\mathbf{w}_{\ell}^{(2)}\right\|_{\mathcal{C}^{0}\left([0, T] ; \mathcal{C}^{1}(\bar{\Omega})\right)^{k}}+\left\|\mathbf{z}_{\ell}^{(2)}\right\|_{\mathcal{C}^{0}\left([0, T] ; \mathcal{C}^{1}(\bar{\Omega})\right)^{m}} \leqslant M,
$$

for some positive $M$. Then there exists a constant $C>0$, depending only on $T, \Omega, \omega, M$ such that we have:

$$
\begin{aligned}
& \left\|\varrho^{(1)}-\bar{\varrho}^{(2)}\right\|_{\left(L^{2}(\Omega)\right)^{N}} \leqslant C\left(\sum_{\ell=1}^{N}\left\|\left(v_{\ell}^{(1)}-v_{\ell}^{(2)}\right)\right\|_{H^{1}\left(0, T ; H^{1}\left(\omega_{0}\right)\right)}\right. \\
& \left.\quad+\left\|\left(v_{\ell}^{(1)}-v_{\ell}^{(2)}\right)\left(\cdot, t_{0}\right)\right\|_{H^{2}(\Omega)}+\left\|\left(\mathbf{w}_{\ell}^{(1)}-\mathbf{w}_{\ell}^{(2)}\right)\left(\cdot, t_{0}\right)\right\|_{L^{2}(\Omega)^{k}}+\left\|\left(\mathbf{z}_{\ell}^{(1)}-\mathbf{z}_{\ell}^{(2)}\right)\left(\cdot, t_{0}\right)\right\|_{L^{2}(\Omega)^{m}}\right),
\end{aligned}
$$

for all $\bar{\varrho}^{(1)} \in \mathcal{A}$. 
Remark 3.1. The condition (3.4) is a straightforward consequence of the two Propositions 2.1 and 2.2 and a Sobolev embedding theorem (e.g., Thm. 5.4 in [1], Cor. 9.1, p. 46, inVol. 1 of [33])

$$
H^{1}\left(0, T ; H^{3}(\Omega)\right) \hookrightarrow \mathcal{C}^{0}\left([0, T] ; \mathcal{C}^{1}(\bar{\Omega})\right),
$$

if the initial conditions verify

$$
v_{\ell}^{(2)}(t=0) \in H^{4}(\Omega), \quad \mathbf{w}_{\ell}^{(2)}(t=0) \in H^{3}(\Omega)^{k}, \quad \text { and } \quad \mathbf{z}_{\ell}^{(2)}(t=0) \in H^{3}(\Omega)^{m} .
$$

Remark 3.2. We imposed a regularity $H^{3}(\Omega)$ in the assumption $(3.2)$, in order to guarantee the $H^{3}$ regularity for the concentration variables $\mathbf{z}$.

By Theorem 3.1, we can readily derive the uniqueness in the inverse problem.

Corollary 3.1. Under the same assumptions as in Theorem 3.1 and if

$$
\begin{gathered}
\left(v_{\ell}^{(1)}\left(x, t_{0}\right), \mathbf{w}_{\ell}^{(1)}\left(x, t_{0}\right), \mathbf{z}_{\ell}^{(1)}\left(x, t_{0}\right)\right)=\left(v_{\ell}^{(2)}\left(x, t_{0}\right), \mathbf{w}_{\ell}^{(2)}\left(x, t_{0}\right), \mathbf{z}_{\ell}^{(2)}\left(x, t_{0}\right)\right), x \in \Omega, \\
v_{\ell}^{(1)}(x, t)=v_{\ell}^{(2)}(x, t), \quad \text { in } \omega \times(0, T),
\end{gathered}
$$

for $\ell=1, \ldots, N$, then $\bar{\varrho}^{(1)}=\bar{\varrho}^{(2)}$ in $\Omega$.

Since the number of the unknown coefficients is $N$, it is natural to expect that $N$-times observations can yield the Lipschitz stability. As is stated in Theorem 3.1. our tool is an $L^{2}$-weighted estimate called Carleman estimate.

As for inverse problems of determining coefficients in parabolic equations, we refer to Elayyan and Isakov [12], Imanuvilov and Yamamoto [20]-[21], Isakov [23], Isakov and Kindermann [24], Ivanchov [25], Klibanov [29], Klibanov and Timonov [30], Yamamoto and Zou [44]. In particular, in [36, 5], determination problems for principal parts are discussed. In those existing papers, the determination of a single coefficient is discussed, while here we consider an inverse problem for the identification of multiple coefficients based on a finite set of observations. Our formulation is with a finite number of observations and this kind of inverse problems was firstly solved by Bukhgeim and Klibanov [7] whose methodology is based on Carleman estimates. For similar inverse problems for other equations, we refer to Bellassoued [3], Bellassoued and Yamamoto [4], Imanuvilov and Yamamoto [21], [22], Isakov [23], Khaidarov [27], Klibanov [28]-[29], Klibanov and Timonov [30], Klibanov and Yamamoto [31], Yamamoto [43].

\section{Global CARLEMAN INEQUALity FOR REACTION-DiFFUSION SYSTEM}

In this section, we give Carleman estimate for the reaction-diffusion model. This Carleman estimate would be used later for the stability and uniqueness of the solution of the parameter identification problem. We are interested in identifying the parameters $\bar{\varrho}_{i}, i=1, \ldots, N$, where $\bar{\varrho}_{i}$ is the maximal conductance associated with the $i^{\text {th }}$ current.

We first have to define the weight function that we will use. This weight is fundamental in the sense that, roughly speaking, information will propagate in space along the gradient lines of this function.

4.1. Weight functions. Let $\omega$ be a subdomain of $\Omega$. We have the following

Lemma 4.1. Let $\omega_{0}$ be an open set such that $\bar{\omega}_{0} \subset \omega$. Then, under the symmetric hypothesis on $\sigma$ and (2.11), there exists a function $\beta \in \mathcal{C}^{2}(\bar{\Omega})$ such that

$$
\beta(x)>0 \quad \forall x \in \Omega, \quad \beta_{\mid \partial \Omega}=0 \quad \text { and } \quad|\nabla \beta(x)|>0 \quad \forall x \in \bar{\Omega} \backslash \omega_{0} .
$$

Moreover, we have

$$
\sigma(x) \nabla \beta(x) \cdot \nu(x) \leqslant 0, \quad x \in \partial \Omega .
$$


The proof of Lemma 4.1] is given in [18].

Remark 1. The construction of the function $\beta$ uses Morse functions and the associated approximation theorem, such a weight function is introduced in [17].

We will now use the function $\beta$ given by Lemma 4.1 to build new weight functions. Let $\lambda$ be a sufficiently large positive constant that only depends on $\Omega$ and $\omega$. For $t \in(0, T)$ we introduce the following functions:

$$
\varphi(x, t)=\frac{e^{\lambda \beta(x)}}{t(T-t)}, \quad \eta(x, t)=\frac{e^{2 \lambda\|\beta\|_{\infty}}-e^{\lambda \beta(x)}}{t(T-t)},
$$

and

$$
\bar{\varphi}(x, t)=\frac{e^{-\lambda \beta(x)}}{t(T-t)}, \quad \bar{\eta}(x, t)=\frac{e^{2 \lambda\|\beta\|_{\infty}}-e^{-\lambda \beta(x)}}{t(T-t)} .
$$

Notice that

$$
\eta(x, t) \leqslant \bar{\eta}(x, t) \quad \forall(x, t) \in Q .
$$

We now have for every $\lambda>0$ the following properties which will be helpful for our calculations

$$
\begin{array}{cc}
\nabla \varphi=\lambda \varphi \nabla \beta, & \nabla \eta=-\lambda \varphi \nabla \beta, \\
\nabla \bar{\varphi}=-\lambda \varphi \nabla \beta, & \nabla \bar{\eta}=\lambda \varphi \nabla \beta . \\
1 \leqslant\left(\frac{T}{2}\right)^{2} \varphi ; \quad \varphi \leqslant\left(\frac{T}{2}\right)^{2} \varphi^{2} ; \quad \varphi \leqslant\left(\frac{T}{2}\right)^{4} \varphi^{3}, \\
\left|\partial_{t} \varphi\right| \leqslant T \varphi^{2}, & \left|\partial_{t t}^{2} \varphi\right| \leqslant 2 T^{2} \varphi^{3}, \\
\left|\partial_{t} \eta\right| \leqslant T \varphi^{2}, & \left|\partial_{t t}^{2} \eta\right| \leqslant 2 T^{2} \varphi^{3} .
\end{array}
$$

We can notice that $\eta$ tends rapidly to $+\infty$ when $t \rightarrow T$ or $t \rightarrow 0$ but that $\eta$ is uniformly bounded in $\Omega \times[\delta, T-\delta]$ if $\delta>0$. Our last weight function will depend on a second parameter $s$ and will be of the form $e^{-s \eta(x, t)}$. We can see that, for fixed $s$, this function tends very rapidly to 0 when $t \rightarrow T$ or $t \rightarrow 0$.

4.2. Global Carleman inequality for parabolic equation. Let $G_{1} \in L^{2}(Q)$ and $u \in H^{1}\left(0, T ; L^{2}(\Omega)\right) \cap$ $L^{2}\left(0, T ; H^{2}(\Omega)\right)$ satisfy the following parabolic equation

$$
\partial_{t} u-\operatorname{div}(\sigma \nabla u)=G_{1}, \quad \text { in } Q
$$

with the boundary condition

$$
\sigma \nabla u \cdot \nu=0, \quad \text { on } \Sigma .
$$

We can now state the global Carleman inequality.

Theorem 4.1. Suppose that $\sigma$ satisfy the condition (2.11). Then, there exists $\lambda_{0}=\lambda_{0}(\Omega, \omega) \geqslant 1, s_{0}=$ $s_{0}\left(\lambda_{0}, T\right)>1$ and a positive constant $C_{0}=C_{0}(\Omega, \omega, T)$ such that, for any $\lambda \geqslant \lambda_{0}$ and any $s \geqslant s_{0}$, the following estimate holds

$$
\begin{aligned}
& \left\|e^{-s \eta}(s \varphi)^{-1 / 2} \partial_{t} u\right\|_{2}^{2}+\left\|e^{-s \eta}(s \varphi)^{-1 / 2} \operatorname{div}(\sigma \nabla u)\right\|_{2}^{2}+s^{3} \lambda^{4}\left\|e^{-s \eta} \varphi^{3 / 2} u\right\|_{2}^{2}+s \lambda^{2}\left\|e^{-s \eta} \varphi^{1 / 2} \nabla u\right\|_{2}^{2} \\
& \quad \leqslant C\left(\left\|e^{-s \eta} G_{1}\right\|_{2}^{2}+s^{3} \lambda^{4} \int_{Q_{\omega}} e^{-2 s \eta} \varphi^{3}|u|^{2} d x d t+s \lambda^{2} \int_{Q_{\omega}} e^{-2 s \eta} \varphi|\nabla u|^{2} d x d t\right),
\end{aligned}
$$

for any $u$ solution to $4.10-(4.11)$ and where $\|\cdot\|_{2}$, is the $L^{2}(Q)-$ norm. 
Proof. The proof of Theorem 4.1 is very much technical. We gives a sketch of proof that is done by steps, following [36].

Step 1. For $s>0$, we define $\psi=e^{-s \eta} u$, we replace in equation (4.10) $u$ by $e^{s \eta} \psi$, and we multiply the equation by $e^{-s \eta}$, we then obtain,

$$
e^{-s \eta}\left(\partial_{t}\left(e^{s \eta} \psi\right)-\operatorname{div}\left(\sigma \nabla\left(e^{s \eta} \psi\right)\right)\right)=e^{-s \eta} G_{1}(x, t) .
$$

By computing (4.13), we obtain an equality with the following symmetric and anti-symmetric operators

$$
L_{1}(\psi(x, t))+L_{2}(\psi(x, t))=F_{1}(\psi(x, t)), \quad(x, t) \in Q,
$$

where

$$
\begin{gathered}
L_{1}(\psi)=s \partial_{t} \eta \psi-s^{2} \lambda^{2} \varphi^{2}|\nabla \beta|_{\sigma}^{2} \psi-\operatorname{div}(\sigma \nabla \psi), \\
L_{2}(\psi)=2 s \lambda^{2} \varphi|\nabla \beta|_{\sigma}^{2} \psi+\partial_{t} \psi+2 s \lambda \varphi \sigma \nabla \beta \nabla \psi, \\
F_{1}(\psi)=e^{-s \eta} G_{1}(x, t)-s \lambda \varphi \operatorname{div}(\sigma \nabla \beta) \psi+s \lambda^{2} \varphi|\nabla \beta|_{\sigma}^{2} \psi .
\end{gathered}
$$

Besides, by virtue of (4.2) and properties of $\eta$ we have

$$
\psi(x, 0)=\psi(x, T)=0
$$

Applying the $L^{2}(Q)$ norm on equation 4.14, we obtain

$$
\left\|L_{1}(\psi)\right\|_{2}^{2}+\left\|L_{2}(\psi)\right\|_{2}^{2}+2\left(L_{1}(\psi), L_{2}(\psi)\right)=\left\|F_{1}(\psi)\right\|_{2}^{2},
$$

where $(.,$.$) denotes the scalar product in L^{2}(Q)$.

In virtue of 4.15) and 4.16, let us compute the scalar product in the left hand side of 4.19), we get

$$
\left(L_{1}(\psi), L_{2}(\psi)\right)=\sum_{i, j=1}^{3} I_{i j} .
$$

In 4.20 , all the terms $I_{i j}$ represent the scalar products of the three terms in $L_{1}(\psi)$ by the three terms in $L_{2}(\psi)$.

Similarely, by defining $\bar{\psi}=e^{-s \bar{\eta}} u$, replacing $u$ by $e^{s \bar{\eta}} \bar{\psi}$ in equation 4.10 and by multiplying the equation by $e^{-s \bar{\eta}}$, we obtain

$$
\bar{L}_{1}(\bar{\psi}(x, t))+\bar{L}_{2}(\bar{\psi}(x, t))=\bar{F}_{1}(\bar{\psi}(x, t)), \quad \text { in }(x, t) \in Q,
$$

where

$$
\begin{gathered}
\bar{L}_{1}(\bar{\psi})=s \partial_{t} \bar{\eta} \bar{\psi}-s^{2} \lambda^{2} \bar{\varphi}^{2}|\nabla \beta|_{\sigma}^{2} \bar{\psi}-\operatorname{div}(\sigma \nabla \bar{\psi}), \\
\bar{L}_{2}(\bar{\psi})=2 s \lambda^{2} \bar{\varphi}|\nabla \beta|_{\sigma}^{2} \bar{\psi}+\partial_{t} \bar{\psi}-2 s \lambda \bar{\varphi} \sigma \nabla \beta \nabla \bar{\psi}, \\
\bar{F}_{1}(\bar{\psi})=e^{-s \bar{\eta}} G_{1}+s \lambda \bar{\varphi} \operatorname{div}(\sigma \nabla \beta) \bar{\psi}+s \lambda^{2} \bar{\varphi}|\nabla \beta|_{\sigma}^{2} \bar{\psi} .
\end{gathered}
$$

Hence, we obtain the scalar products

$$
\left(\bar{L}_{1}(\bar{\psi}), \bar{L}_{2}(\bar{\psi})\right)=\sum_{i, j=1}^{3} \bar{I}_{i j} .
$$

In 4.25 , all the terms $\bar{I}_{i j}$ represent the scalar products of the three terms in $\bar{L}_{1}(\bar{\psi})$ by the three terms in $\bar{L}_{2}(\psi)$. 
Step 2. In the sequel, by $C$ we mean various constants independent of $s, \lambda$ and $T$ as we want to keep track of the powers of $s, \lambda$ and $T$ involved. In order to organize the calculations, we will give particular importance to terms

$$
J_{1}=s^{3} \lambda^{4} \int_{Q} \varphi^{3}|\psi|^{2} d x d t, \quad \text { and } \quad J_{2}=s \lambda^{2} \int_{Q} \varphi|\nabla \psi|^{2} d x d t
$$

From the definitions of $\varphi, \bar{\varphi}, \eta$ and $\bar{\eta}$ we have

$$
\bar{\varphi} \leqslant \varphi, \quad \eta \leqslant \bar{\eta}, \quad \text { and } \quad|\bar{\psi}| \leqslant|\psi|, \quad \text { in } Q .
$$

Additionally, since we have $\bar{\psi}=e^{-s(\bar{\eta}-\eta)} \psi$, we obtain

$$
|\nabla \bar{\psi}| \leqslant C(|\nabla \psi|+s \lambda \varphi|\nabla \beta||\bar{\psi}|) \leqslant C(|\nabla \psi|+s \lambda \varphi|\psi|)
$$

where the constant $c$ depends only on $\beta$.

In addition, due to hypothesis on $\beta$ of Lemma 4.1, we use that

$$
\begin{aligned}
\left|\partial_{i} \sigma_{i j} \sigma_{k l} \partial_{k} \beta\right| & \leqslant C(\Omega, \omega), \\
\left|\sigma_{i j} \partial_{j}\left(\sigma_{k l} \partial_{k} \beta\right)\right| & \leqslant C(\Omega, \omega), \\
|\operatorname{div}(\sigma \nabla \beta)| & \leqslant C(\Omega, \omega), \\
\left|\sigma_{k l} \partial_{l} \sigma_{i j} \partial_{k} \beta\right| & \leqslant C(\Omega, \omega), \\
\left|\sigma_{i j} \partial_{j} \beta \partial_{i} \beta\right| & \leqslant C(\Omega, \omega) .
\end{aligned}
$$

Using Green formula, some terms $I_{i, j}$ and $\bar{I}_{i, j}$ would be absorbed by $J_{1}$ and $J_{2}$, then by summing them and using the fondamental properties of the function $\beta$ given by Lemma 4.1, we obtain

$$
\begin{aligned}
\left\|L_{1}(\psi)\right\|_{2}^{2}+\left\|L_{2}(\psi)\right\|_{2}^{2} & +s^{3} \lambda^{4} \int_{Q} \varphi^{3}|\psi|^{2} d x d t+s \lambda^{2} \int_{Q} \varphi|\nabla \psi|^{2} d x d t \\
& \leqslant 2\left\|e^{-s \eta} G_{1}\right\|_{2}^{2}+C\left(s^{3} \lambda^{4} \int_{Q_{\omega}} \varphi^{3}|\psi|^{2} d x d t+s \lambda^{2} \int_{Q_{\omega}} \varphi|\nabla \psi|^{2} d x d t\right) .
\end{aligned}
$$

Step 3. Back to the original variable $u$, we deduce the result of Theorem 4.1 .

This complete the proof of 4.12 .

In order to prove Theorem 3.1, we need the following lemma to estimate the gaiting variable $\mathrm{w}$ and the ionic concentration $\mathbf{z}$.

Lemma 4.2. Let $t_{0}=T / 2$. There exists $C>0$ such that the following estimate

$$
\int_{Q} e^{-2 s \eta(x, t)}|\mathbf{u}(x, t)|^{2} d x d t \leqslant C\left(\int_{Q} e^{-2 s \eta(x, t)}\left|\mathbf{u}\left(x, t_{0}\right)\right|^{2} d x d t+\frac{1}{s} \int_{Q} e^{-2 s \eta(x, t)}\left|\partial_{t} \mathbf{u}(x, t)\right|^{2} d x d t\right),
$$

holds for any $\mathbf{u} \in H^{1}\left(0, T ; L^{2}(\Omega)\right)$ and any $s>0$.

Proof. By Cauchy-Schwarz inequality, we obtain

$$
\int_{Q}\left|\int_{t_{0}}^{t} \partial_{t} \mathbf{u}(x, \tau) d \tau\right|^{2} e^{-2 s \eta(x, t)} d x d t \leqslant \int_{Q}\left(\int_{t_{0}}^{t}\left|\partial_{t} \mathbf{u}(x, \tau)\right|^{2} d \tau\right)\left(t-t_{0}\right) e^{-2 s \eta(x, t)} d x d t
$$

Using the fact that

$$
\partial_{t} \eta(x, t)=\frac{2\left(t-t_{0}\right)}{t^{2}(T-t)^{2}}\left(e^{2 \lambda\|\beta\|_{\infty}}-e^{\lambda \beta}\right),
$$


then, we get

$$
\begin{array}{r}
\int_{Q}\left|\int_{t_{0}}^{t} \partial_{t} \mathbf{u}(x, \tau) d \tau\right|^{2} e^{-2 s \eta(x, t)} d x d t \leqslant C \\
\int_{Q}\left(\int_{t_{0}}^{t}\left|\partial_{t} \mathbf{u}(x, \tau)\right|^{2} d \tau\right) \partial_{t} \eta(x, t) e^{-2 s \eta(x, t)} d x d t \\
=-\frac{C}{2 s} \int_{Q}\left(\int_{t_{0}}^{t}\left|\partial_{t} \mathbf{u}(x, \tau)\right|^{2} d \tau\right) \partial_{t}\left(e^{-2 s \eta(x, t)}\right) d x d t
\end{array}
$$

By noting that $e^{-2 s \eta(x, T)}=e^{-2 s \eta(x, 0)}=0$, the integration by parts with respect to the time variable implies that the right hand side of 4.36 is equal to

$$
\frac{C}{2 s} \int_{Q}\left|\partial_{t} \mathbf{u}(x, t)\right|^{2} e^{-2 s \eta(x, t)} d x d t
$$

We write

$$
|\mathbf{u}(x, t)|^{2} \leqslant C\left(\left|\mathbf{u}(x, t)-\mathbf{u}\left(x, t_{0}\right)\right|^{2}+\left|\mathbf{u}\left(x, t_{0}\right)\right|^{2}\right),
$$

then, we deduce that

$$
\begin{array}{rl}
\int_{Q} e^{-2 s \eta(x, t)}|\mathbf{u}(x, t)|^{2} & d x d t \leqslant C \int_{Q} e^{-2 s \eta(x, t)}\left(\left|\int_{t_{0}}^{t} \partial_{t} \mathbf{u}(x, \tau) d \tau\right|^{2}+\left|\mathbf{u}\left(x, t_{0}\right)\right|^{2}\right) d x d t \\
& \leqslant C\left(\int_{Q} e^{-2 s \eta(x, t)}\left|\mathbf{u}\left(x, t_{0}\right)\right|^{2} d x d t+\frac{1}{s} \int_{Q} e^{-2 s \eta(x, t)}\left|\partial_{t} \mathbf{u}(x, t)\right|^{2} d x d t\right) .
\end{array}
$$

Thus, the proof of Lemma 4.2 is completed.

\section{STABILITY ESTIMATE OF CONDUCTANCES PARAMETERS}

This section is devoted to proof Theorem 3.1. The idea of the proof is based on the Carleman estimate methode.

5.1. Linearized problem. In this section, we discuss a linearized inverse problem of determining $\bar{\varrho}$. We consider the solutions $\left(v^{(n)}, \mathbf{w}^{(n)}, \mathbf{z}^{(n)}\right), n=1,2$, to the following systems

$$
\begin{cases}\partial_{t} v^{(n)}-\operatorname{div}\left(\sigma \nabla v^{(n)}\right)=I_{a p p}+I_{i o n}\left(\varrho^{(n)}, v^{(n)}, \mathbf{w}^{(n)}, \mathbf{z}^{(n)}\right) & \text { in } Q, \\ \partial_{t} \mathbf{w}^{(n)}=\boldsymbol{F}\left(v^{(n)}, \mathbf{w}^{(n)}\right) & \text { in } Q, \\ \partial_{t} \mathbf{z}^{(n)}=\boldsymbol{G}\left(\bar{\varrho}^{(n)}, v^{(n)}, \mathbf{w}^{(n)}, \mathbf{z}^{(n)}\right) & \text { in } Q, \\ \sigma \nabla v^{(n)} \cdot \nu=0 & \text { on } \Sigma,\end{cases}
$$

and we consider the difference

$$
v=v^{(1)}-v^{(2)}, \quad \mathbf{w}=\mathbf{w}^{(1)}-\mathbf{w}^{(2)}, \quad \mathbf{z}=\mathbf{z}^{(1)}-\mathbf{z}^{(2)}, \quad \bar{\varrho}=\bar{\varrho}^{(1)}-\bar{\varrho}^{(2)} .
$$

Then, $(v, \mathbf{w}, \mathbf{z})$ is solution to the following problem

$$
\begin{cases}\partial_{t} v-\operatorname{div}(\sigma \nabla v)=h\left(\bar{\varrho}^{(1)}, \bar{\varrho}^{(2)}, v^{(1)}, \mathbf{w}^{(1)}, \mathbf{z}^{(1)}, v^{(2)}, \mathbf{w}^{(2)}, \mathbf{z}^{(2)}\right) & \text { in } Q \\ \partial_{t} \mathbf{w}=\Phi\left(v^{(1)}, \mathbf{w}^{(1)}, v^{(2)}, \mathbf{w}^{(2)}\right) & \text { in } Q \\ \partial_{t} \mathbf{z}=\Psi\left(v^{(1)}, \mathbf{w}^{(1)}, \mathbf{z}^{(1)}, v^{(2)}, \mathbf{w}^{(2)}, \mathbf{z}^{(2)}\right) & \text { in } Q \\ \sigma \nabla v \cdot \nu=0 & \text { on } \Sigma .\end{cases}
$$


Here

(5.4)

$$
\begin{aligned}
h\left(\varrho^{(1)}, \bar{\varrho}^{(2)}, v^{(1)}, \mathbf{w}^{(1)}, \mathbf{z}^{(1)}, v^{(2)}, \mathbf{w}^{(2)}, \mathbf{z}^{(2)}\right) & =I_{\text {ion }}\left(\bar{\varrho}^{(1)}, v^{(1)}, \mathbf{w}^{(1)}, \mathbf{z}^{(1)}\right)-I_{\text {ion }}\left(\bar{\varrho}^{(2)}, v^{(2)}, \mathbf{w}^{(2)}, \mathbf{z}^{(2)}\right) \\
& =\mathbf{S}^{\top}\left(v^{(2)}, \mathbf{w}^{(2)}, \mathbf{z}^{(2)}\right) \cdot \varrho+R\left(v^{(1)}, \mathbf{w}^{(1)}, \mathbf{z}^{(1)}, v^{(2)}, \mathbf{w}^{(2)}, \mathbf{z}^{(2)}\right),
\end{aligned}
$$

where $\mathbf{S}=\left(S_{i}\right)_{1 \leqslant i \leqslant N}$ with

$$
S_{i}\left(v^{(2)}, \mathbf{w}^{(2)}, \mathbf{z}^{(2)}\right)=y_{i}\left(v^{(2)}\right)\left(v^{(2)}-E_{i}\left(\mathbf{z}^{(2)}\right)\right) \prod_{j=1}^{k}\left(w_{j}^{(2)}\right)^{p_{j, i}}, \quad i=1, \ldots, N, \quad \mathbf{w}^{(2)}=\left(w_{1}^{(2)}, \ldots, w_{k}^{(2)}\right),
$$

and

$$
R\left(v^{(1)}, \mathbf{w}^{(1)}, \mathbf{z}^{(1)}, v^{(2)}, \mathbf{w}^{(2)}, \mathbf{z}^{(2)}\right)=I_{i o n}\left(\bar{\varrho}^{(1)}, v^{(1)}, \mathbf{w}^{(1)}, \mathbf{z}^{(1)}\right)-I_{i o n}\left(\bar{\varrho}^{(1)}, v^{(2)}, \mathbf{w}^{(2)}, \mathbf{z}^{(2)}\right) .
$$

In $(5.4),{ }^{\top}$ represent the transpose of any matrix and the expression $\mathbf{S}^{\top}\left(v^{(2)}, \mathbf{w}^{(2)}, \mathbf{z}^{(2)}\right) \cdot \bar{\varrho}$ is the Euclidian scalar products of the row vector $\mathbf{S}^{\top}$ and the colon vector $\bar{\varrho}:=\left(\bar{\varrho}_{i}\right)_{1 \leqslant i \leqslant N}$ formed by the ionic parameters $\varrho_{i}$.

Finally the functions $\Phi$ and $\Psi$ are respectively given by

$$
\begin{aligned}
\Phi\left(v^{(1)}, \mathbf{w}^{(1)}, v^{(2)}, \mathbf{w}^{(2)}\right) & =\boldsymbol{F}\left(v^{(1)}, \mathbf{w}^{(1)}\right)-\boldsymbol{F}\left(v^{(2)}, \mathbf{w}^{(2)}\right), \\
\Psi\left(v^{(1)}, \mathbf{w}^{(1)}, \mathbf{z}^{(1)}, v^{(2)}, \mathbf{w}^{(2)}, \mathbf{z}^{(2)}\right) & =\boldsymbol{G}\left(\bar{\varrho}^{(1)}, v^{(1)}, \mathbf{w}^{(1)}, \mathbf{z}^{(1)}\right)-\boldsymbol{G}\left(\bar{\varrho}^{(2)}, v^{(2)}, \mathbf{w}^{(2)}, \mathbf{z}^{(2)}\right) .
\end{aligned}
$$

In the next we denote

$$
R(x, t)=R\left(v^{(1)}, \mathbf{w}^{(1)}, \mathbf{z}^{(1)}, v^{(2)}, \mathbf{w}^{(2)}, \mathbf{z}^{(2)}\right)(x, t), \quad \mathbf{S}(x, t)=\mathbf{S}^{\top}\left(v^{(2)}, \mathbf{w}^{(2)}, \mathbf{z}^{(2)}\right)(x, t),
$$

and

$$
h(x, t)=h\left(\varrho^{(1)}, \bar{\varrho}^{(2)}, v^{(1)}, \mathbf{w}^{(1)}, \mathbf{z}^{(1)}, v^{(2)}, \mathbf{w}^{(2)}, \mathbf{z}^{(2)}\right):=\mathbf{S}^{\top}(x, t) \cdot \bar{\varrho}(x)+R(x, t) .
$$

Let $p=\partial_{t} v$ and we consider the time derivative of the first equation of the system (5.3)

$$
\partial_{t} p-\operatorname{div}(\sigma \nabla p)=f \quad \text { in } Q,
$$

with the boundary condition

$$
\sigma \nabla p \cdot \nu=0 \quad \text { on } \Sigma
$$

where

$$
f(x, t)=\partial_{t} h(x, t)=\partial_{t} \mathbf{S}^{\top}(x, t) \cdot \bar{\varrho}(x)+\partial_{t} R(x, t), \quad(x, t) \in Q .
$$

The vector functions $\mathbf{S}^{\top}$ and the function $R$ are defined in 5.5 and 5.6 respectively. First, we evaluate the first equation (5.11) at a fixed time $t_{0}$ such that $2 t_{0}=T$

$$
p\left(x, t_{0}\right)-\operatorname{div}\left(\sigma \nabla v\left(x, t_{0}\right)\right)-\mathbf{S}^{\top}\left(x, t_{0}\right) \cdot \bar{\varrho}-R\left(x, t_{0}\right)=0 .
$$

Then, we integrate over $\Omega$ the square of 5.14 with the weight function $e^{-2 s \eta\left(x, t_{0}\right)}$, we obtain

$$
\begin{aligned}
& \int_{\Omega} e^{-2 s \eta\left(x, t_{0}\right)}\left|\mathbf{S}^{\top}\left(x, t_{0}\right) \cdot \bar{\varrho}(x)\right|^{2} d x \leqslant C\left(\int_{\Omega} e^{-2 s \eta\left(x, t_{0}\right)}\left|p\left(x, t_{0}\right)\right|^{2} d x\right. \\
& \left.+\int_{\Omega} e^{-2 s \eta\left(x, t_{0}\right)}\left|\operatorname{div}\left(\sigma \nabla v\left(x, t_{0}\right)\right)\right|^{2} d x+\int_{\Omega} e^{-2 s \eta\left(x, t_{0}\right)}\left|R\left(x, t_{0}\right)\right|^{2} d x\right) \\
& \leqslant C\left(\int_{\Omega} e^{-2 s \eta\left(x, t_{0}\right)}\left|p\left(x, t_{0}\right)\right|^{2} d x+\int_{\Omega} e^{-2 s \eta\left(x, t_{0}\right)}\left|R\left(x, t_{0}\right)\right|^{2} d x+\left\|v\left(\cdot, t_{0}\right)\right\|_{H^{2}(\Omega)}^{2}\right) .
\end{aligned}
$$

In order to estimate the second term in the RHS of (5.15), we need the following lemma: 
Lemma 5.1. Let $\left(v^{(n)}, \mathbf{w}^{(n)}, \mathbf{z}^{(n)}\right), n=1,2$, the solutions of $(5.1)$. Then there exist a constant $C>0$ such that the following estimates hold

$$
|R(x, t)|^{2} \leqslant C\left(|v(x, t)|^{2}+|\mathbf{w}(x, t)|^{2}+|\mathbf{z}(x, t)|^{2}\right),
$$

and

$$
\left|\partial_{t} R(x, t)\right|^{2} \leqslant C \sum_{s=0}^{1}\left(\left|\partial_{t}^{s} v(x, t)\right|^{2}+\left|\partial_{t}^{s} \mathbf{w}(x, t)\right|^{2}+\left|\partial_{t}^{s} \mathbf{z}(x, t)\right|^{2}\right) .
$$

Here, $(v, \mathbf{w}, \mathbf{z})$ is given by (5.2).

Proof. By a computation, we have

$$
\begin{aligned}
R(x, t) & =\sum_{i=1}^{N} \bar{\varrho}_{i}^{(1)} \prod_{j=1}^{k}\left(w_{j}^{(2)}\right)^{p_{j, i}}\left(y_{i}\left(v^{(1)}\right) v^{(1)}-y_{i}\left(v^{(2)}\right) v^{(2)}\right) \\
& +\sum_{i=1}^{N} \bar{\varrho}_{i}^{(1)}\left(\sum_{s=1}^{\max _{j} p_{j, i}} \sum_{|\alpha|=s} \frac{\left(\mathbf{w}^{(1)}-\mathbf{w}^{(2)}\right)^{\alpha}}{\alpha !} \mathcal{P}_{i, \alpha}\left(\mathbf{w}^{(2)}\right)\right) y_{i}\left(v^{(1)}\right) v^{(1)} \\
& +\sum_{i=1}^{N} \bar{\varrho}_{i}^{(1)}\left(\prod_{j=1}^{k}\left(w_{j}^{(1)}\right)^{p_{j, i}} y_{i}\left(v^{(1)}\right)\left(E_{i}\left(\mathbf{z}^{(2)}\right)-E_{i}\left(\mathbf{z}^{(1)}\right)\right)\right) \\
& -\sum_{i=1}^{N} \bar{\varrho}_{i}^{(1)}\left(\sum_{s=1}^{\max _{j} p_{j, i}} \sum_{|\alpha|=s} \frac{\left(\mathbf{w}^{(1)}-\mathbf{w}^{(2)}\right)^{\alpha}}{\alpha !} \mathcal{P}_{i, \alpha}\left(\mathbf{w}^{(2)}\right)\right) y_{i}\left(v^{(2)}\right) E_{i}\left(\mathbf{z}^{(2)}\right) \\
& -\sum_{i=1}^{N} \bar{\varrho}_{i}^{(1)} \prod_{j=1}^{k}\left(w_{j}^{(1)}\right)^{p_{j, i}}\left(y_{i}\left(v^{(1)}\right)-y_{i}\left(v^{(2)}\right)\right) E_{i}\left(\mathbf{z}^{(2)}\right),
\end{aligned}
$$

where

$$
\mathcal{P}_{i, \alpha}(\xi)=\frac{\partial^{|\alpha|}}{\partial \xi^{\alpha}}\left(\prod_{j=1}^{k} \xi_{j}^{p_{j, i}}\right), \quad \xi=\left(\xi_{1}, \ldots, \xi_{k}\right) \in \mathbb{R}^{k}
$$

By (5.18), we have

$$
\begin{aligned}
|R(x, t)|^{2} \leqslant C \sum_{i=1}^{N} & \left(\left|y_{i}\left(v^{(1)}\right) v^{(1)}-y_{i}\left(v^{(2)}\right) v^{(2)}\right|^{2}+\left|y_{i}\left(v^{(1)}\right)-y_{i}\left(v^{(2)}\right)\right|^{2}\right. \\
& \left.+\sum_{s=1}^{\max _{j} p_{j, i}}\left|\mathbf{w}^{(1)}-\mathbf{w}^{(2)}\right|^{2 s}+\left|E_{i}\left(\mathbf{z}^{(2)}\right)-E_{i}\left(\mathbf{z}^{(1)}\right)\right|^{2}\right) .
\end{aligned}
$$

Back to estimate (5.16), we can show that

$$
\begin{array}{r}
\left|E_{i}\left(\mathbf{z}^{(2)}\right)-E_{i}\left(\mathbf{z}^{(1)}\right)\right|^{2}=\left|\bar{\gamma}_{i}\right|\left|\log \left(\frac{z_{e}}{z_{i}^{(2)}}\right)-\log \left(\frac{z_{e}}{z_{i}^{(1)}}\right)\right|^{2} \\
\leqslant C\left|\log \left(z_{i}^{(1)}\right)-\log \left(z_{i}^{(2)}\right)\right|^{2} \leqslant C\left|z_{i}\right|^{2}
\end{array}
$$

and

$$
\left|y_{i}\left(v^{(1)}\right) v^{(1)}-y_{i}\left(v^{(2)}\right) v^{(2)}\right|^{2} \leqslant C|v|^{2},
$$


since $z \mapsto \log z$ and $v \mapsto y_{i}(v) v$ are Lipschitz functions. Then

$$
|R(x, t)|^{2} \leqslant C\left(|v(x, t)|^{2}+\sum_{s=1}^{\max _{j} p_{j, i}}|\mathbf{w}(x, t)|^{2 s}+|\mathbf{z}(x, t)|^{2}\right) .
$$

Using the fact that $\left|w_{j}(x, t)\right| \leqslant 1$, we deduce

$$
|R(x, t)|^{2} \leqslant C\left(|v(x, t)|^{2}+|\mathbf{w}(x, t)|^{2}+|\mathbf{z}(x, t)|^{2}\right) .
$$

To prove inequality (5.17), we use

$$
\begin{aligned}
&\left|\partial_{t} R\right|^{2} \leqslant C \sum_{i=1}^{N}\left(\left|y_{i}\left(v^{(1)}\right) v^{(1)}-y_{i}\left(v^{(2)}\right) v^{(2)}\right|^{2}\right.+\left|\partial_{t}\left(y_{i}\left(v^{(1)}\right) v^{(1)}-y_{i}\left(v^{(2)}\right) v^{(2)}\right)\right|^{2}+\left|y_{i}\left(v^{(1)}\right)-y_{i}\left(v^{(2)}\right)\right|^{2} \\
&+\left|\partial_{t}\left(y_{i}\left(v^{(1)}\right)-y_{i}\left(v^{(2)}\right)\right)\right|^{2}+\sum_{s=1}^{\max _{j} p_{j, i}}\left|\mathbf{w}^{(1)}-\mathbf{w}^{(2)}\right|^{2 s}+\left|\partial_{t}\left(\sum_{s=1}^{\max _{j} p_{j, i}} \sum_{|\boldsymbol{\alpha}|=s}\left(\mathbf{w}^{(1)}-\mathbf{w}^{(2)}\right){ }^{\alpha}\right)\right|^{2} \\
&\left.+\left|E_{i}\left(\mathbf{z}^{(1)}\right)-E_{i}\left(\mathbf{z}^{(2)}\right)\right|^{2}+\left|\partial_{t}\left(E_{i}\left(\mathbf{z}^{(2)}\right)-E_{i}\left(\mathbf{z}^{(1)}\right)\right)\right|^{2}\right) .
\end{aligned}
$$

By (2.13, 2.14) and Lemma 2.2, we deduce that

$$
\begin{aligned}
& \left|\partial_{t}\left(E_{i}\left(\mathbf{z}^{(1)}\right)-E_{i}\left(\mathbf{z}^{(2)}\right)\right)\right|^{2}=\left|\bar{\gamma}_{i}\right|\left|\partial_{t}\left(\log z_{i}^{(1)}-\log z_{i}^{(2)}\right)\right|^{2} \\
& =\left|\bar{\gamma}_{i}\right|\left|\frac{\partial_{t} z_{i}^{(1)}}{z_{i}^{(1)}}-\frac{\partial_{t} z_{i}^{(2)}}{z_{i}^{(2)}}\right|^{2} \\
& =\left|\bar{\gamma}_{i}\right|\left|\frac{\partial_{t}\left(z_{i}^{(1)}-z_{i}^{(2)}\right)}{z_{i}^{(1)}}+\frac{\partial_{t} z_{i}^{(2)}}{z_{i}^{(1)}}-\frac{\partial_{t} z_{i}^{(2)}}{z_{i}^{(2)}}\right|^{2} \\
& =\left|\bar{\gamma}_{i}\right|\left|\frac{\partial_{t} z_{i}}{z_{i}^{(1)}}-\frac{z_{i} \partial_{t} z_{i}^{(2)}}{z_{i}^{(1)} z_{i}^{(2)}}\right|^{2} \\
& \leqslant C\left(\left|\partial_{t} z_{i}\right|^{2}+\left|z_{i}\right|^{2}\right)
\end{aligned}
$$

and

$$
\begin{aligned}
\left|\partial_{t}\left(\sum_{s=1}^{\max _{j} p_{j, i}} \sum_{|\alpha|=s}\left(\mathbf{w}^{(1)}-\mathbf{w}^{(2)}\right)^{\alpha}\right)\right|^{2} & =\left|\sum_{s=1}^{\max _{j} p_{j, i}} \sum_{|\alpha|=s} \partial_{t}\left(\prod_{j=1}^{k}\left(w_{j}^{(1)}-w_{j}^{(2)}\right)^{\alpha_{j}}\right)\right|^{2} \\
& \leqslant C \sum_{s=1}^{\max _{j} p_{j, i}} \sum_{|\alpha|=s} \sum_{j=1}^{k}\left|\partial_{t}\left(w_{j}^{(1)}-w_{j}^{(2)}\right)\right|^{2} \\
& \leqslant C\left|\partial_{t} \mathbf{w}\right|^{2}
\end{aligned}
$$

and

$$
\begin{aligned}
& \left|\partial_{t}\left(y_{i}\left(v^{(1)}\right) v^{(1)}-y_{i}\left(v^{(2)}\right) v^{(2)}\right)\right|^{2}=\left|\partial_{t} v^{(1)}\left(y_{i}^{\prime}\left(v^{(1)}\right) v^{(1)}+y_{i}\left(v^{(1)}\right)\right)-\partial_{t} v^{(2)}\left(y_{i}^{\prime}\left(v^{(2)}\right) v^{(2)}+y_{i}\left(v^{(2)}\right)\right)\right|^{2} \\
& =\left(\partial_{t} v^{(1)}-\partial_{t} v^{(2)}\right)\left(y_{i}^{\prime}\left(v^{(1)}\right) v^{(1)}+y_{i}\left(v^{(1)}\right)\right) \\
& +\left.\partial_{t} v^{(2)}\left(y_{i}^{\prime}\left(v^{(1)}\right) v^{(1)}-y_{i}^{\prime}\left(v^{(2)}\right) v^{(2)}+y_{i}\left(v^{(1)}\right)-y_{i}\left(v^{(2)}\right)\right)\right|^{2} \\
& \leqslant C\left(|v|^{2}+\left|\partial_{t} v\right|^{2}\right) \text {. }
\end{aligned}
$$


Here, we have used that $y_{i}(v)$ is a $C^{\infty}$ function with respect to the variable $v$ and then is locally Lipschitz since $v$ is bounded. Similarly for the function $y_{i}(v) v$. The proof is complete.

We integrate on $\Omega \sqrt{5.16}$ for $t=t_{0}$ with the weight function $e^{-2 s \eta\left(x, t_{0}\right)}$, we obtain

$$
\begin{aligned}
\int_{\Omega} e^{-2 s \eta\left(x, t_{0}\right)}\left|R\left(x, t_{0}\right)\right|^{2} d x \leqslant C\left(\left\|e^{-s \eta\left(x, t_{0}\right)} v\left(x, t_{0}\right)\right\|_{L^{2}(\Omega)}^{2}+\right. & \left\|e^{-s \eta\left(x, t_{0}\right)} \mathbf{w}\left(x, t_{0}\right)\right\|_{L^{2}(\Omega)^{k}}^{2} \\
& \left.+\left\|e^{-s \eta\left(x, t_{0}\right)} \mathbf{z}\left(x, t_{0}\right)\right\|_{L^{2}(\Omega)^{m}}^{2}\right) .
\end{aligned}
$$

and we deduce the following Lemma.

Lemma 5.2. Let $\left(v^{(n)}, \mathbf{w}^{(n)}, \mathbf{z}^{(n)}\right), n=1,2$, the solutions of $(5.1)$. Then there exist a constant $C>0$ such that the following estimates hold

$$
\int_{\Omega} e^{-2 s \eta\left(x, t_{0}\right)}\left|R\left(x, t_{0}\right)\right|^{2} d x \leqslant C\left(\left\|v\left(x, t_{0}\right)\right\|_{L^{2}(\Omega)}^{2}+\left\|\mathbf{w}\left(x, t_{0}\right)\right\|_{L^{2}(\Omega)^{k}}^{2}+\left\|\mathbf{z}\left(x, t_{0}\right)\right\|_{L^{2}(\Omega)^{m}}^{2}\right),
$$

for any $s>0$.

The next part is devoted to estimate the first term in the RHS of (5.15).

Lemma 5.3. Let $\omega_{0}$ be an open subdomain of $\Omega$. There exists constants $\lambda_{*}, s_{*}$ and $C>0$ such that for any $s \geqslant s_{*}$ and any $\lambda \geqslant \lambda_{*}$, the following estimate holds

$$
\int_{\Omega} e^{-2 s \eta\left(x, t_{0}\right)}\left|p\left(x, t_{0}\right)\right|^{2} d x \leqslant \frac{C}{s}\left\|e^{-s \eta} f\right\|_{L^{2}(Q)}^{2}+C \lambda^{2} \int_{Q_{\omega_{0}}} e^{-2 s \eta}\left(s^{2} \varphi^{3}|p|^{2}+\varphi|\nabla p|^{2}\right) d x d t
$$

Proof. We have

$$
\begin{array}{r}
K_{1}=\int_{\Omega} e^{-2 s \eta\left(x, t_{0}\right)}\left|p\left(x, t_{0}\right)\right|^{2} d x=\int_{0}^{t_{0}} \int_{\Omega} \frac{d}{d t}\left(e^{-2 s \eta(x, t)}|p|^{2}\right) d x d t \\
=\int_{0}^{t_{0}} \int_{\Omega}\left(-2 s \partial_{t} \eta(x, t)|p|^{2}+2 p \partial_{t} p\right) e^{-2 s \eta(x, t)} d x d t .
\end{array}
$$

Besides, from (4.9) and using Young inequality, we have

$$
\begin{aligned}
\left.\left|-2 s \partial_{t} \eta(x, t)\right| p\right|^{2}+2 p \partial_{t} p \mid & \leqslant 2 s\left|\partial_{t} \eta(x, t)\right||p|^{2}+2|p|\left|\partial_{t} p\right| \\
& \leqslant C\left(s \varphi^{2}|p|^{2}+2\left(s \varphi^{1 / 2}|p|\right)\left(s^{-1} \varphi^{-1 / 2}\left|\partial_{t} p\right|\right)\right. \\
& \leqslant C\left(s \varphi^{2}|p|^{2}+s^{2} \varphi|p|^{2}+s^{-2} \varphi^{-1}\left|\partial_{t} p\right|^{2}\right) \\
& \leqslant C\left(s^{2} \varphi^{2}|p|^{2}+s^{-2} \varphi^{-1}\left|\partial_{t} p\right|^{2}\right) .
\end{aligned}
$$

Then, we apply the Carleman inequality given in Theorem 4.1 satisfied by $p$, we obtain that for $s$ and $\lambda$ sufficiently large

$$
K_{1} \leqslant \frac{C}{s}\left\|e^{-s \eta} f\right\|_{L^{2}(Q)}^{2}+C\left(s^{2} \lambda^{4} \int_{Q_{\omega_{0}}} e^{-2 s \eta} \varphi^{3}|p|^{2} d x d t+\lambda^{2} \int_{Q_{\omega_{0}}} e^{-2 s \eta} \varphi|\nabla p|^{2} d x d t\right) .
$$

This completes the proof.

Lemma 5.4. There exist $C>0$ such that the following estimate

$$
\left\|e^{-s \eta} f\right\|_{L^{2}(Q)}^{2} \leqslant C\left(\int_{Q} e^{-2 s \eta}\left(\left|\partial_{t} \mathbf{S}^{\top} \cdot \bar{\varrho}\right|^{2}+|v|^{2}+|p|^{2}+|\mathbf{w}|^{2}+\left|\partial_{t} \mathbf{w}\right|^{2}+|\mathbf{z}|^{2}+\left|\partial_{t} \mathbf{z}\right|^{2}\right) d x d t\right),
$$

holds for any $s>0$. 
Proof. Using the definition of $f=\sum_{i=1}^{N}\left(\partial_{t} \mathbf{S}\right)_{i}^{\top} \bar{\varrho}_{i}+\partial_{t} R$, and taking into account 5.17, we get

$$
\left\|e^{-s \eta} f\right\|_{L^{2}(Q)}^{2} \leqslant C \int_{Q} e^{-2 s \eta}\left(\left|\partial_{t} \mathbf{S}^{\top} \cdot \bar{\varrho}\right|^{2}+|v|^{2}+|p|^{2}+|\mathbf{w}|^{2}+\left|\partial_{t} \mathbf{w}\right|^{2}+|\mathbf{z}|^{2}+\left|\partial_{t} \mathbf{z}\right|^{2}\right) d x d t
$$

The proof is complete.

Lemma 5.5. There exists constants $\lambda_{*}, s_{*}$ and $C>0$ such that for any $s \geqslant s_{*}$ and any $\lambda \geqslant \lambda_{*}$, the following estimate holds

$$
\begin{aligned}
& \left\|e^{-s \eta} f\right\|_{L^{2}(Q)}^{2} \leqslant C\left(\int_{Q} e^{-2 s \eta}\left(\left|\partial_{t} \mathbf{S}^{\top} \cdot \bar{\varrho}\right|^{2}+\left|\mathbf{S}^{\top} \cdot \bar{\varrho}\right|^{2}+|\bar{\varrho}|^{2}\right) d x d t\right. \\
& \quad+s \lambda^{2} \int_{Q_{\omega_{0}}} e^{-2 s \eta}\left(s^{2} \lambda^{2} \varphi^{3}|v|^{2}+\varphi|\nabla v|^{2}\right) d x d t \\
& \left.\quad+\lambda^{2} \int_{Q_{\omega_{0}}} e^{-2 s \eta}\left(s^{-2} \varphi|\nabla p|^{2}+\lambda^{2} \varphi^{3}|p|^{2}\right) d x d t+\left\|\mathbf{w}\left(., t_{0}\right)\right\|_{L^{2}(\Omega)^{k}}^{2}+\left\|\mathbf{z}\left(., t_{0}\right)\right\|_{L^{2}(\Omega)^{m}}^{2}\right) .
\end{aligned}
$$

Proof. We apply again the Carleman inequality given in Theorem 4.1 satisfied by $p=\partial_{t} v$,

$$
\int_{Q} e^{-2 s \eta}|p|^{2} d x d t \leqslant C\left(s^{-3}\left\|e^{-s \eta} f\right\|_{L^{2}(Q)}^{2}+\lambda^{2} \int_{Q_{\omega_{0}}} e^{-2 s \eta}\left(\lambda^{2} \varphi^{3}|p|^{2}+s^{-2} \varphi|\nabla p|^{2}\right) d x d t\right) .
$$

Let us now estimate the following terms

$$
I_{1}=\int_{Q} e^{-2 s \eta}\left|\partial_{t} \mathbf{w}\right|^{2} d x d t \quad \text { and } \quad I_{2}=\int_{Q} e^{-2 s \eta}\left|\partial_{t} \mathbf{z}\right|^{2} d x d t .
$$

From system (5.3) and (5.7), we have

$$
\partial_{t} w_{j}=\Phi_{j}\left(v^{(1)}, w_{j}^{(1)}, v^{(2)}, w_{j}^{(2)}\right)=F_{j}\left(v^{(1)}, w_{j}^{(1)}\right)-F_{j}\left(v^{(2)}, w_{j}^{(2)}\right), \quad \forall j=1, \ldots, k .
$$

Since the function $F_{j}: \mathbb{R}^{2} \rightarrow \mathbb{R}$ is locally Lipschitz continuous, we have

$$
I_{1}=\int_{Q} e^{-2 s \eta}\left|\partial_{t} \mathbf{w}\right|^{2} d x d t \leqslant C \int_{Q} e^{-2 s \eta}\left(|v|^{2}+|\mathbf{w}|^{2}\right) d x d t
$$

Now, from (5.8) and 2.7), we have

$$
\begin{aligned}
& \left|\partial_{t} z_{i}\right|^{2} \leqslant C\left(\left|H_{i}\left(v^{(1)}, \mathbf{w}^{(1)}, \mathbf{z}^{(1)}\right)-H_{i}\left(v^{(2)}, \mathbf{w}^{(2)}, \mathbf{z}^{(2)}\right)\right|^{2}\right. \\
& \left.+\left|J_{i}\left(\bar{\varrho}^{1}, v^{(1)}, \mathbf{w}^{(1)}, \log z_{i}^{(1)}\right)-J_{i}\left(\bar{\varrho}^{2}, v^{(2)}, \mathbf{w}^{(2)}, \log z_{i}^{(2)}\right)\right|^{2}\right) \\
& \quad \leqslant C\left(|v|^{2}+|\mathbf{w}|^{2}+|\mathbf{z}|^{2}+|\bar{\varrho}|^{2}\right),
\end{aligned}
$$

since $v^{j} \in L^{\infty}(Q), \mathbf{w}^{(2)} \in L^{\infty}(Q)^{k}, \log \mathbf{z}^{(2)} \in L^{\infty}(Q)^{m}$, and the fact that the ionic curent $J_{i}$ is locally Lipschitz. Then

$$
I_{2}=\int_{Q} e^{-2 s \eta}\left|\partial_{t} \mathbf{z}\right|^{2} d x d t \leqslant C \int_{Q} e^{-2 s \eta}\left(|v|^{2}+|\mathbf{w}|^{2}+|\mathbf{z}|^{2}+|\bar{\varrho}|^{2}\right) d x d t .
$$


Substituting (5.35), 5.37), 5.39) in 5.33, we obtain

$$
\begin{aligned}
\left\|e^{-s \eta} f\right\|_{L^{2}(Q)}^{2} \leqslant C\left(\int _ { Q } e ^ { - 2 s \eta } \left(\left|\partial_{t} \mathbf{S}^{\top} \cdot \bar{\varrho}\right|^{2}\right.\right. & \left.|\bar{\varrho}|^{2}+|v|^{2}+|\mathbf{w}|^{2}+|\mathbf{z}|^{2}\right) d x d t \\
& \left.+\lambda^{2} \int_{Q_{\omega_{0}}} e^{-2 s \eta}\left(s^{-2} \varphi|\nabla p|^{2}+\lambda^{2} \varphi^{3}|p|^{2}\right) d x d t\right) .
\end{aligned}
$$

Using Lemma 4.2, we get

$$
\int_{Q} e^{-2 s \eta}|\mathbf{w}|^{2} d x d t \leqslant C \int_{Q} e^{-2 s \eta}\left(\left|\mathbf{w}\left(x, t_{0}\right)\right|^{2}+s^{-1}|\Phi(x, t)|^{2}\right) d x d t .
$$

Using the Carleman inequality given by Theorem 4.1 for the estimation of $|v|^{2}$, using lemma 4.2 for $|\mathbf{z}|^{2}$ and the estimation 5.41, we get, for $s$ and $\lambda$ large enough

$$
\begin{array}{r}
\left\|e^{-s \eta} f\right\|_{L^{2}(Q)}^{2} \leqslant C\left(\int_{Q} e^{-2 s \eta}\left(|h|^{2}+|\Phi|^{2}+|\Psi|^{2}\right) d x d t+\left\|\mathbf{w}\left(., t_{0}\right)\right\|_{L^{2}(\Omega)^{k}}^{2}+\left\|\mathbf{z}\left(., t_{0}\right)\right\|_{L^{2}(\Omega)^{m}}^{2}\right. \\
+s \lambda^{2} \int_{Q_{\omega_{0}}} e^{-2 s \eta}\left(s^{2} \lambda^{2} \varphi^{3}|v|^{2}+\varphi|\nabla v|^{2}\right) d x d t+\lambda^{2} \int_{Q_{\omega_{0}}} e^{-2 s \eta}\left(s^{-2} \varphi|\nabla p|^{2}+\lambda^{2} \varphi^{3}|p|^{2}\right) d x d t \\
\left.+\int_{Q} e^{-2 s \eta}\left(\left|\partial_{t} \mathbf{S}^{\top} \cdot \bar{\varrho}\right|^{2}+|\varrho|^{2}\right) d x d t\right) .
\end{array}
$$

Setting $D:=\int_{Q} e^{-2 s \eta}\left(|h|^{2}+|\Phi|^{2}+|\Psi|^{2}\right) d x d t$, and using the estimates 5.37, 5.39, we obtain, for $s$ and $\lambda$ large enough

$$
D \leqslant C \int_{Q} e^{-2 s \eta}\left(\left|\mathbf{S}^{\top} \cdot \bar{\varrho}\right|^{2}+|v|^{2}+|\mathbf{w}|^{2}+|\mathbf{z}|^{2}\right) d x d t
$$

since $v^{j} \in L^{\infty}(Q), \mathbf{w}^{(2)} \in L^{\infty}(Q)^{k}, \log \mathbf{z}^{(2)} \in L^{\infty}(Q)^{m}$. Using again the Carleman inequality given by Theorem 4.1 to $|v|^{2}$ and using Lemma 4.2 to $|\mathbf{w}|^{2}$, we get, for $s$ and $\lambda$ large enough

$$
\begin{array}{r}
D \leqslant C \int_{Q} e^{-2 s \eta}\left(\left|\mathbf{S}^{\top} \cdot \bar{\varrho}\right|^{2}+|\bar{\varrho}|^{2}\right) d x d t+C\left(\lambda^{2} \int_{Q_{\omega_{0}}} e^{-2 s \eta}\left(\lambda^{2} \varphi^{3}|v|^{2}+s^{-2} \varphi|\nabla v|^{2}\right) d x d t\right) \\
+\left\|\mathbf{w}\left(., t_{0}\right)\right\|_{L^{2}(\Omega)^{k}}^{2}+\left\|\mathbf{z}\left(., t_{0}\right)\right\|_{L^{2}(\Omega)^{m}}^{2}
\end{array}
$$

Substituting (5.44) in (5.42), we obtain

$$
\begin{array}{r}
\left\|e^{-s \eta} f\right\|_{L^{2}(Q)}^{2} \leqslant C\left(\int_{Q} e^{-2 s \eta}\left(\left|\mathbf{S}^{\top} \cdot \bar{\varrho}\right|^{2}+|\bar{\varrho}|^{2}+\left|\partial_{t} \mathbf{S}^{\top} \cdot \bar{\varrho}\right|^{2}\right) d x d t+s^{3} \lambda^{4} \int_{Q_{\omega_{0}}} e^{-2 s \eta} \varphi^{3}|v|^{2} d x d t\right. \\
+s \lambda^{2} \int_{Q_{\omega_{0}}} e^{-2 s \eta} \varphi|\nabla v|^{2} d x d t+s^{-2} \lambda^{2} \int_{Q_{\omega_{0}}} e^{-2 s \eta} \varphi|\nabla p|^{2} d x d t+\lambda^{4} \int_{Q_{\omega_{0}}} e^{-2 s \eta} \varphi^{3}|p|^{2} d x d t \\
\left.+\left\|\mathbf{w}\left(., t_{0}\right)\right\|_{L^{2}(\Omega)^{k}}^{2}+\left\|\mathbf{z}\left(., t_{0}\right)\right\|_{L^{2}(\Omega)^{m}}^{2}\right) .
\end{array}
$$

This complete the proof. 
5.2. End of the proof of Theorem 3.1. Then, inserting these above inequalities $(5.28),(5.29)$ and (5.34) in (5.15), it yields

$$
\begin{aligned}
\int_{\Omega} e^{-2 s \eta\left(x, t_{0}\right)}\left|\mathbf{S}^{\top}\left(x, t_{0}\right) \cdot \bar{\varrho}(x)\right|^{2} d x \leqslant & C\left(s^{-1} \int_{Q} e^{-2 s \eta}|\bar{\varrho}(x)|^{2} d x d t+\|v\|_{H^{1}\left(0, T ; H^{1}\left(\omega_{0}\right)\right)}^{2}\right. \\
& \left.+\left\|v\left(\cdot, t_{0}\right)\right\|_{H^{2}(\Omega)}^{2}+\left\|\mathbf{w}\left(\cdot, t_{0}\right)\right\|_{L^{2}(\Omega)^{k}}^{2}+\left\|\mathbf{z}\left(\cdot, t_{0}\right)\right\|_{L^{2}(\Omega)^{m}}^{2}\right) .
\end{aligned}
$$

Since $v_{\ell}^{(2)} \in W^{1, \infty}(Q), \mathbf{w}_{\ell}^{(2)} \in L^{\infty}(Q)^{k}$ and $\log \mathbf{z}_{\ell}^{2} \in L^{\infty}(Q)^{m}, \ell=1, \ldots, N$. Summing up the above estimate over $\ell=1, \ldots, N$, we get that

$$
\begin{aligned}
& \int_{\Omega} e^{-2 s \eta\left(x, t_{0}\right)}|\Lambda(x) \cdot \bar{\varrho}(x)|^{2} d x \leqslant C\left(N s^{-1} \int_{\Omega} e^{-2 s \eta\left(x, t_{0}\right)}|\bar{\varrho}(x)|^{2} d x\right. \\
& \left.+\sum_{\ell=1}^{N}\left(\left\|v_{\ell}\right\|_{H^{1}\left(0, T ; H^{1}\left(\omega_{0}\right)\right)}^{2}+\left\|v_{\ell}\left(., t_{0}\right)\right\|_{H^{2}(\Omega)}^{2}+\left\|\mathbf{w}_{\ell}\left(., t_{0}\right)\right\|_{L^{2}(\Omega)^{k}}^{2}+\left\|\mathbf{z}_{\ell}\left(., t_{0}\right)\right\|_{L^{2}(\Omega)^{m}}^{2}\right)\right),
\end{aligned}
$$

where the $N \times N$ real matrix $\Lambda(x)=\Lambda\left(v_{\ell}^{(2)}\left(x, t_{0}\right), \mathbf{w}_{\ell}^{(2)}\left(x, t_{0}\right), \mathbf{z}_{\ell}^{(2)}\left(x, t_{0}\right)\right)$, for $x \in \Omega$. Notice that we have

$$
\|\Lambda(x) \xi\|_{\mathbb{R}^{N}} \geqslant \alpha_{1}(x)|\xi|_{\mathbb{R}^{N}}, \quad x \in \Omega, \quad \xi \in \mathbb{R}^{N},
$$

where $\left(\alpha_{j}(x)\right)_{1 \leqslant j \leqslant N} \subset \mathbb{R}_{+}^{N}$ denotes the increasing sequence of the singular values of $\Lambda(x)$, and $|\xi|_{\mathbb{R}^{N}}$ stands for the Euclidian norm of $\xi$.

Moreover, by Sobolev embedding theorem (e.g., Thm. 5.4 in [1], Cor. 9.1, p. 46, inVol. 1 of [33]), we see that $H^{3}(\Omega) \subset \mathcal{C}^{1}(\bar{\Omega})$, hence $v_{\ell}^{(2)}\left(., t_{0}\right), \mathbf{w}_{\ell}^{(2)}\left(., t_{0}\right)$ and $\log \mathbf{z}_{\ell}^{(2)}\left(., t_{0}\right), \ell=1, \ldots, N$, being taken in $\mathcal{C}^{1}(\bar{\Omega})$ thanks to Proposition 2.2. Then, $\alpha_{1} \in \mathcal{C}^{1}\left(\bar{\Omega} ; \mathbb{R}_{+}\right)$from [26] [Thm 6.8 p. 122]. This combined with equation (3.4), yields $\alpha_{0}:=\inf _{x \in \Omega} \alpha_{1}(x)>0$. As a Consequence and by [5.48), we have

$$
\int_{\Omega} e^{-2 s \eta\left(x, t_{0}\right)}|\Lambda(x) \bar{\varrho}(x)|^{2} d x \geqslant \alpha_{0}^{2} \int_{\Omega} e^{-2 s \eta\left(x, t_{0}\right)}|\bar{\varrho}(x)|^{2} d x
$$

and Theorem 3.1 follows directly from this and 5.46 by choosing $s$ so large that $C N s^{-1}<\alpha_{0}^{2}$.

\section{DisCUSSION AND CONCLUSIONS}

Accurate mathematical models are a necessary step towards the development of personalized cardiac models from a set of observed data. Most of the accurate and physiologically-detailed models are based on the $\mathrm{HH}$ formalism in which the ionic current is scaled by conductance parameters. Thus, in model personalization procedures, identifying the conductance parameters is a key point in order to be able to accurately assimilate the data. These parameters act directly on the ionic current representing the reaction term in the PDE. They also act directly on the concentration rate and implicitly on the gate variables. In this work, we proved that the parameter identification inverse problem is stable under certain conditions. These conditions have to be satisfied in order to proceed to the computational estimation. Unless, there in no guarantee about the uniqueness of the parameters obtained in the numerical results. Our approach is based on a new Carleman inequality for the monodomain reaction diffusion model coupled to a general form of ordinary differential equation system. We followed the same procedure as in [36, 5] dealing with much simpler phenomenological models. But here, the Carleman inequality that we established for the ODE system was adapted to the formalism of the physiological ionic models. This Carleman inequality is crucial in order to prove the global Carleman estimate for nonlinear parabolic equation coupled with the ordinary differential system, and thus, for solving the parameter identification stability problem. Here we addressed the problem of identifiying ion channels conductances but the same strategy could be used for the other 
parameters of the transmembrane potential model. This result is very important step in order to numerically solve the parameters identification problem in cardiac electrophysiology because it provides the condition in which this problem is stable. The same approach could be used for other applications in electrophysiology such as models in cerebral, intestine and uterine electrical activity. There are still some limitations that could be addressed in order to reach the conditions of real life applications. The first issue is that it not possible in practice to measure observation in a subset volume of the 3D domain. Ideally observations should be considered on a part of the accessible boundary of the domain. The second issue is that not all of the state variables are mesurable in real life application. In general, only the electrical potential is mesurable in clinical application. In future works, our aim is to try to solve these two open questions.

\section{APPENDICES}

We will make use of the following Gronwall lemma.

\section{Lemma 7.1. (Gronwall's lemma)}

Let $\gamma \in \mathbb{R}, \phi \in \mathcal{C}^{1}([0, T], \mathbb{R})$ and $f \in \mathcal{C}^{0}([0, T], \mathbb{R})$ with

$$
\phi^{\prime}(t) \leqslant \gamma \phi(t)+f(t)
$$

then

$$
\forall t \in[0, T], \quad \phi(t) \leqslant e^{\gamma t} \phi(0)+\int_{0}^{t} e^{\gamma(t-s)} f(s) d s .
$$

Proof of Proposition 2.1.

First we take the time derivative of equations system (2.8)

$$
\begin{cases}\partial_{t t} v-\operatorname{div}\left(\boldsymbol{\sigma} \nabla \partial_{t} v\right)=\partial_{t} I_{\text {app }}+\partial_{t} I_{i o n} & \text { in } Q \equiv \Omega \times(0, T), \\ \partial_{t t} \mathbf{w}=\partial_{t} \boldsymbol{F}(v, \mathbf{w}) & \text { in } Q, \\ \partial_{t t} \mathbf{z}=\partial_{t} \boldsymbol{G}(\varrho, v, \mathbf{w}, \mathbf{z}) & \text { in } Q,\end{cases}
$$

with initial condition

$$
\begin{aligned}
\partial_{t} v(t=0) & =\operatorname{div}\left(\boldsymbol{\sigma} \nabla v_{0}\right)+I_{a p p}(t=0)+I_{i o n}(t=0) & & \text { in } \Omega, \\
\partial_{t} \mathbf{w}(t=0) & =\boldsymbol{F}\left(v_{0}, w_{0}\right) & & \text { in } \Omega, \\
\partial_{t} \mathbf{z}(t=0) & =\boldsymbol{G}\left(\bar{\varrho}, v_{0}, \mathbf{w}_{0}, \mathbf{z}_{0}\right) & & \text { in } \Omega .
\end{aligned}
$$

Using the hypothesis $v_{0} \in H^{2}(\Omega), \mathbf{w}_{0} \in L^{2}(\Omega)^{k}$ and $\mathbf{z}_{0} \in L^{2}(\Omega)^{m}$, we deduce that

$$
\operatorname{div}\left(\boldsymbol{\sigma} \nabla v_{0}\right) \in L^{2}(\Omega), \quad I_{\text {ion }}(t=0) \in L^{2}(\Omega),
$$

and then $\partial_{t} v(t=0) \in L^{2}(\Omega)$ since $I_{a p p}$ satisfy the hypothesis $\sqrt{2.9}$. Also we deduce that

$$
\partial_{t} \mathbf{w}(t=0) \in L^{2}(\Omega)^{k}, \quad \text { and } \quad \partial_{t} \mathbf{z}(t=0) \in L^{2}(\Omega)^{m} .
$$

We integrate over $\Omega$ the sum of the first equation of 7.2 multiplied by $\partial_{t} v$, apply the divergence theorem, and use the BC that $\sigma \nabla \partial_{t} v \cdot \nu=0$ on $\partial \Omega$, and of the second one multiplied by $\partial_{t} \mathbf{w}$ and the third one multiplied by $\partial_{t} \mathbf{z}$, we obtain

$$
\begin{aligned}
& \frac{1}{2} \frac{d}{d t}\left(\left\|\partial_{t} v\right\|_{L^{2}(\Omega)}^{2}+\left\|\partial_{t} \mathbf{w}\right\|_{L^{2}(\Omega)^{k}}^{2}+\left\|\partial_{t} \mathbf{z}\right\|_{L^{2}(\Omega)^{m}}^{2}\right)+\int_{\Omega} \boldsymbol{\sigma} \nabla \partial_{t} v \cdot \nabla \partial_{t} v d x= \\
& \int_{\Omega} \partial_{t} v \partial_{t} I_{a p p} d x+\int_{\Omega} \partial_{t} v \partial_{t} I_{\text {ion }} d x+\int_{\Omega} \partial_{t} \mathbf{w} \cdot \partial_{t} \boldsymbol{F} d x+\int_{\Omega} \partial_{t} \mathbf{z} \cdot \partial_{t} \boldsymbol{G} d x
\end{aligned}
$$


First, from 2.11 we consider

$$
\int_{\Omega} \boldsymbol{\sigma} \nabla \partial_{t} v \cdot \nabla \partial_{t} v d x \geqslant \mu \int_{\Omega}\left|\nabla \partial_{t} v\right|^{2} d x=\mu\left\|\nabla \partial_{t} v\right\|_{L^{2}(\Omega)}^{2}
$$

Second, from Cauchy-Schwarz inequality and Young inequality, we have

$$
\int_{\Omega} \partial_{t} v \partial_{t} I_{a p p} d x \leqslant \frac{1}{2}\left(\left\|\partial_{t} v\right\|_{L^{2}(\Omega)}^{2}+\left\|\partial_{t} I_{a p p}\right\|_{L^{2}(\Omega)}^{2}\right),
$$

since $I_{a p p}$ verify the condition 2.9 and thanks to Lemma 2.1, we have $\partial_{t} v \in L^{2}(\Omega)$.

We write

$$
\begin{aligned}
\partial_{t} v \partial_{t} I_{i o n}= & \sum_{i=1}^{N} \bar{\varrho}_{i}\left|\partial_{t} v\right|^{2} y_{i}^{\prime}(v) \prod_{j=1}^{k} w_{j}^{p_{j, i}}\left(v-E_{i}(\mathbf{z})\right) \\
& +\sum_{i=1}^{N} \bar{\varrho}_{i} \partial_{t} v y_{i}(v)\left(\sum_{l=1}^{k} p_{l, i} w_{l}^{p_{l, i}-1} \partial_{t} w_{l} \prod_{j \neq l} w_{j}^{p_{j, i}}\right)\left(v-E_{i}(\mathbf{z})\right) \\
& +\sum_{i=1}^{N} \bar{\varrho}_{i}\left|\partial_{t} v\right|^{2} y_{i}(v) \prod_{j=1}^{k} w_{j}^{p_{j, i}}+\sum_{i=1}^{N} \bar{\varrho}_{i} \partial_{t} v \frac{\partial_{t} z_{i}}{z_{i}} y_{i}(v) \prod_{j=1}^{k} w_{j}^{p_{j, i}} .
\end{aligned}
$$

Thanks to Lemma 2.1, we have $v \in L^{\infty}(Q), \log \mathbf{z} \in L^{\infty}(Q)^{m}, \mathbf{w} \in[0,1]^{k}$ and $y_{i}$ is $\mathcal{C}^{\infty}$. Applying Cauchy-Schwarz inequality and Young inequality, we deduce from (7.8) that

$$
\int_{\Omega} \partial_{t} v \partial_{t} I_{\text {app }} d x+\int_{\Omega} \partial_{t} v \partial_{t} I_{i o n} d x \leqslant C\left(\left\|\partial_{t} v\right\|_{L^{2}(\Omega)}^{2}+\left\|\partial_{t} \mathbf{w}\right\|_{L^{2}(\Omega)^{k}}^{2}+\left\|\partial_{t} \mathbf{z}\right\|_{L^{2}(\Omega)^{m}}^{2}+\left\|\partial_{t} I_{\text {app }}\right\|_{L^{2}(\Omega)}^{2}\right) .
$$

We recall that the function $F_{j}$ given by $[2.3)$ is $\mathcal{C}^{2}\left(\mathbb{R}^{2}\right)$ for $j=1, \ldots, k$, and then we can write

$$
\partial_{t} F_{j}\left(v, w_{j}\right)=\partial_{t} v \partial_{1} F_{j}+\partial_{t} w_{j} \partial_{2} F_{j}
$$

where $\partial_{l}$ is the partial derivative with respect the $l^{t h}$ variable, $l=1,2$. Since $\alpha_{j}, \beta_{j}$ are $\mathcal{C}^{\infty}(\mathbb{R}), v \in L^{\infty}(Q)$ and $w_{j} \in[0,1], j=1, \ldots, k$, we deduce that

(7.12) $\partial_{1} F_{j}\left(v, w_{j}\right)=\alpha^{\prime}(v)\left(1-w_{j}\right)-\beta^{\prime}(v) w_{j}, \quad$ and $\quad \partial_{2} F_{j}\left(v, w_{j}\right)=-\alpha(v)-\beta(v), \quad j=1, \ldots, k$, are bounded. Then, by Cauchy-Schwarz inequality and Young inequality, we have

$$
\int_{\Omega} \partial_{t} \mathbf{w} \cdot \partial_{t} \boldsymbol{F} d x \leqslant C\left(\left\|\partial_{t} v\right\|_{L^{2}(\Omega)}^{2}+\left\|\partial_{t} \mathbf{w}\right\|_{L^{2}(\Omega)^{k}}^{2}\right) .
$$

Similarly for the variable $\mathbf{z}$, for $i=1, \ldots, m$, we can write

$$
\begin{aligned}
\partial_{t} G_{i}(\bar{\varrho}, v, \mathbf{w}, \mathbf{z})= & -\left(\partial_{t} v \partial_{2} J_{i}+\sum_{j=1}^{k}\left(\partial_{t} w_{j} \partial_{j+2} J_{i}\right)+\partial_{t} \log z_{i} \partial_{k+3} J_{i}\right) \\
& +\partial_{t} v \partial_{2} H_{i}+\sum_{j=1}^{k} \partial_{t} w_{j} \partial_{j+2} H_{i}+\sum_{j=1}^{m} \partial_{t} \log z_{j} \partial_{k+2+j} H_{i} .
\end{aligned}
$$

By hypothesis 2.6 and 2.7), we deduce that $\partial_{l} J_{i}, l=1, \ldots, k+3$, and $\partial_{l} H_{i}, l=1, \ldots, m+k+2$, are bounded since $v \in L^{\infty}(Q), \mathbf{w} \in[0,1]^{k}, \mathbf{z} \in L^{\infty}(Q)^{m}$ and from Lemma 2.2. Then we have

$$
\int_{\Omega} \partial_{t} \mathbf{z} \cdot \partial_{t} \boldsymbol{G} d x \leqslant C\left(\left\|\partial_{t} v\right\|_{L^{2}(\Omega)}^{2}+\left\|\partial_{t} \mathbf{w}\right\|_{L^{2}(\Omega)^{k}}^{2}+\left\|\partial_{t} \mathbf{z}\right\|_{L^{2}(\Omega)^{m}}^{2}\right)
$$


Thus, substituting (7.7), (7.10), (7.13) and (7.15) in (7.6), we obtain

(7.16)

$$
\begin{gathered}
\frac{d}{d t}\left(\left\|\partial_{t} v\right\|_{L^{2}(\Omega)}^{2}+\left\|\partial_{t} \mathbf{w}\right\|_{L^{2}(\Omega)^{k}}^{2}+\left\|\partial_{t} \mathbf{z}\right\|_{L^{2}(\Omega)^{m}}^{2}\right)+\mu\left\|\nabla \partial_{t} v\right\|_{L^{2}(\Omega)}^{2} \leqslant \\
C\left(\left\|\partial_{t} v\right\|_{L^{2}(\Omega)}^{2}+\left\|\partial_{t} \mathbf{w}\right\|_{L^{2}(\Omega)^{k}}^{2}+\left\|\partial_{t} \mathbf{z}\right\|_{L^{2}(\Omega)^{m}}^{2}+\left\|\partial_{t} I_{a p p}\right\|_{L^{2}(\Omega)}^{2}\right) .
\end{gathered}
$$

Applying Gronwall Lemma (Lemma 7.1), we deduce

$$
\left\|\partial_{t} v\right\|_{L^{2}(\Omega)}^{2}+\left\|\partial_{t} \mathbf{w}\right\|_{L^{2}(\Omega)^{k}}^{2}+\left\|\partial_{t} \mathbf{z}\right\|_{L^{2}(\Omega)^{m}}^{2}+\mu\left\|\nabla \partial_{t} v\right\|_{L^{2}(\Omega)}^{2} \leqslant C_{0}, \quad \forall t \in[0, T],
$$

where

$$
C_{0}=C\left(\left\|v_{0}\right\|_{H^{2}(\Omega)},\left\|\mathbf{w}_{0}\right\|_{L^{2}(\Omega)^{k}},\left\|\mathbf{z}_{0}\right\|_{L^{2}(\Omega)^{m}},\left\|I_{a p p}\right\|_{H^{1}\left(0, T ; L^{2}(\Omega)\right)}\right)>0 .
$$

Thus,

$$
v \in W^{1, \infty}\left(0, T ; H^{1}(\Omega)\right), \quad \mathbf{w} \in W^{1, \infty}\left(0, T ; L^{2}(\Omega)\right)^{k}, \quad \text { and } \quad \mathbf{z} \in W^{1, \infty}\left(0, T ; L^{2}(\Omega)\right)^{m} .
$$

Now, for $t \in(0, T)$, we intergrate 7.16 over $(0, t)$, we obtain

$$
\left\|\partial_{t} v\right\|_{L^{2}(\Omega)}^{2}+\left\|\partial_{t} \mathbf{w}\right\|_{L^{2}(\Omega)^{k}}^{2}+\left\|\partial_{t} \mathbf{z}\right\|_{L^{2}(\Omega)^{m}}^{2}+\mu \int_{0}^{t}\left\|\nabla \partial_{t} v\right\|_{L^{2}(\Omega)}^{2} d \tau \leqslant 2 C_{0}
$$

and then

$$
\int_{0}^{T}\left\|\nabla \partial_{t} v\right\|_{L^{2}(\Omega)}^{2} d \tau \leqslant 2 C_{0} .
$$

We conclude

$$
v \in H^{1}\left(0, T ; H^{1}(\Omega)\right) .
$$

On the other hand, we write

$$
\operatorname{div}(\boldsymbol{\sigma} \nabla v)=\partial_{t} v-I_{a p p}-I_{i o n} \in L^{\infty}\left(0, T ; L^{2}(\Omega)\right),
$$

then

$$
v \in L^{\infty}\left(0, T ; H^{2}(\Omega)\right) .
$$

Let us now consider the hypothesis $v_{0} \in H^{3}(\Omega)$ and $(2.23)$. We have

$$
\nabla\left(\partial_{t} v(t=0)\right)=\nabla\left(\operatorname{div} \nabla v_{0}\right)+\nabla I_{a p p}(t=0)+\nabla I_{i o n}\left(v_{0}, \mathbf{w}_{0}, \mathbf{z}_{0}\right) \in L^{2}(\Omega) .
$$

We multiply the first (respectively second, third) equation of $(7.2)$ by $\partial_{t t} v$ (respectively $\partial_{t t} \mathbf{w}, \partial_{t t} \mathbf{z}$ ), we obtain

$$
\begin{aligned}
& \left|\partial_{t t} v\right|^{2}-\partial_{t t} v \operatorname{div}\left(\boldsymbol{\sigma} \nabla \partial_{t} v\right)=\partial_{t t} v \partial_{t} I_{a p p}+\partial_{t t} v \partial_{t} I_{i o n}, \\
& \left|\partial_{t t} w_{j}\right|^{2}+\frac{1}{2}(\alpha(v)+\beta(v)) \frac{d}{d t}\left|\partial_{t} w_{j}\right|^{2}=\partial_{t t} w_{j} \partial_{t} v \partial_{1} F_{j}, \quad j=1, \cdots, k, \\
& \left|\partial_{t t} z_{i}\right|^{2}+\frac{1}{2 z_{i}} \partial_{k+3} J_{i} \frac{d}{d t}\left|\partial_{t} z_{i}\right|^{2}=\partial_{t t} z_{i}\left(-\partial_{t} v \partial_{2} J_{i}-\sum_{j=1}^{k} \partial_{t} w_{j} \partial_{j+2} J_{i}\right. \\
& \left.+\partial_{t} v \partial_{2} H_{i}+\sum_{j=1}^{k} \partial_{t} w_{j} \partial_{j+2} H_{i}+\sum_{j=1}^{m} \partial_{t} \log z_{j} \partial_{k+2+j} H_{i}\right), \quad i=1, \cdots, m .
\end{aligned}
$$


We integrate over $\Omega$, using Cauchy-Schwarz inequality and Young inequality, for $j=1, \ldots, k$ and $i=$ $1, \ldots, m$, we have

(7.27)

$$
\begin{aligned}
& \left\|\partial_{t t} v\right\|_{L^{2}(\Omega)}^{2}+\frac{d}{d t}\left|\nabla \partial_{t} v\right|_{\sigma}^{2} \leqslant \varepsilon\left\|\partial_{t t} v\right\|_{L^{2}(\Omega)}^{2}+C_{\varepsilon}\left(\left\|\partial_{t} I_{a p p}\right\|_{L^{2}(\Omega)}^{2}+\left\|\partial_{t} I_{i o n}\right\|_{L^{2}(\Omega)}^{2}\right) \\
& \left\|\partial_{t t} w_{j}\right\|_{L^{2}(\Omega)}^{2}+C_{\alpha, \beta} \frac{d}{d t}\left\|\partial_{t} w_{j}\right\|_{L^{2}(\Omega)}^{2} \leqslant \varepsilon\left\|\partial_{t t} w_{j}\right\|_{L^{2}(\Omega)}^{2}+C_{\varepsilon}\left\|\partial_{t t} v\right\|_{L^{2}(\Omega)}^{2}, \\
& \left\|\partial_{t t} z_{i}\right\|_{L^{2}(\Omega)}^{2}+C_{i} \frac{d}{d t}\left\|\partial_{t} z_{i}\right\|_{L^{2}(\Omega)}^{2} \leqslant \varepsilon\left\|\partial_{t t} z_{i}\right\|_{L^{2}(\Omega)}^{2}+C_{\varepsilon}\left(\left\|\partial_{t} v\right\|_{L^{2}(\Omega)}^{2}+\left\|\partial_{t} \mathbf{w}\right\|_{L^{2}(\Omega)^{k}}^{2}+\left\|\partial_{t} z_{i}\right\|_{L^{2}(\Omega)}^{2}\right) .
\end{aligned}
$$

Here we have used two contants $C_{\alpha, \beta}, C_{i}>0$ such that $\alpha(v)+\beta(v) \geqslant C_{\alpha, \beta}$ and $\frac{1}{z_{i}} \partial_{k+3} J_{i} \geqslant C_{i}$.

Summing all equations in 7.27

$$
\begin{gathered}
\left\|\partial_{t t} v\right\|_{L^{2}(\Omega)}^{2}+\left\|\partial_{t t} \mathbf{w}\right\|_{L^{2}(\Omega)^{k}}^{2}+\left\|\partial_{t t} \mathbf{z}\right\|_{L^{2}(\Omega)^{m}}^{2}+\frac{d}{d t}\left(\left|\nabla \partial_{t} v\right|_{\boldsymbol{\sigma}}^{2}+\left\|\partial_{t} \mathbf{w}\right\|_{L^{2}(\Omega)^{k}}^{2}+\left\|\partial_{t} \mathbf{z}\right\|_{L^{2}(\Omega)^{m}}^{2}\right) \leqslant \\
C\left(\left\|\partial_{t} I_{\text {app }}\right\|_{L^{2}(\Omega)}^{2}+\left\|\partial_{t} I_{i o n}\right\|_{L^{2}(\Omega)}^{2}+\left\|\partial_{t} v\right\|_{L^{2}(\Omega)}^{2}+\left\|\partial_{t} \mathbf{w}\right\|_{L^{2}(\Omega)^{k}}^{2}+\left\|\partial_{t} \mathbf{z}\right\|_{L^{2}(\Omega)^{m}}^{2}\right) .
\end{gathered}
$$

Using hypothesis $(7.25)$, for $t \in[0, T]$, we integrate over the time interval $(0, t)$

(7.29)

$$
\begin{array}{r}
\int_{0}^{t}\left(\left\|\partial_{t t} v\right\|_{L^{2}(\Omega)}^{2}+\left\|\partial_{t t} \mathbf{w}\right\|_{L^{2}(\Omega)^{k}}^{2}+\left\|\partial_{t t} \mathbf{z}\right\|_{L^{2}(\Omega)^{m}}^{2}\right) d \tau+\mu\left\|\nabla \partial_{t} v\right\|_{L^{2}(\Omega)}^{2}+\left\|\partial_{t} \mathbf{w}\right\|_{L^{2}(\Omega)^{k}}^{2}+\left\|\partial_{t} \mathbf{z}\right\|_{L^{2}(\Omega)^{m}}^{2} \leqslant \\
C\left(\left\|\partial_{t} I_{\text {app }}\right\|_{L^{2}\left(0, T ; L^{2}(\Omega)\right)}^{2}+\left\|\partial_{t} I_{\text {ion }}\right\|_{L^{2}\left(0, T ; L^{2}(\Omega)\right)}^{2}+C C_{0} T+C_{\boldsymbol{\sigma}}\left\|\nabla \partial_{t} v(t=0)\right\|_{L^{2}(\Omega)}^{2}\right) .
\end{array}
$$

Here $C_{\boldsymbol{\sigma}}$ is a non negative constant such that $\left|\nabla \partial_{t} v(t=0)\right|_{\boldsymbol{\sigma}} \leqslant C_{\boldsymbol{\sigma}}\left\|\nabla \partial_{t} v(t=0)\right\|_{L^{2}(\Omega)}$ and $C_{0}$ is given by (7.18).

We deduce

$$
\int_{0}^{T}\left(\left\|\partial_{t t} v\right\|_{L^{2}(\Omega)}^{2}+\left\|\partial_{t t} \mathbf{w}\right\|_{L^{2}(\Omega)^{k}}^{2}+\left\|\partial_{t t} \mathbf{z}\right\|_{L^{2}(\Omega)^{m}}^{2}\right)+\left\|\nabla \partial_{t} v\right\|_{L^{2}(\Omega)}^{2}+\left\|\partial_{t} \mathbf{w}\right\|_{L^{2}(\Omega)^{k}}^{2}+\left\|\partial_{t} \mathbf{z}\right\|_{L^{2}(\Omega)^{m}}^{2} \leqslant C t e
$$

then

$$
v \in H^{2}\left(0, T ; L^{2}(\Omega)\right), \quad \mathbf{w} \in H^{2}\left(0, T ; L^{2}(\Omega)\right)^{k}, \quad \text { and } \quad \mathbf{z} \in H^{2}\left(0, T ; L^{2}(\Omega)\right)^{m} .
$$

Let us now prove the regularities (2.25). Deriving equations (2.3) and (2.5) over the space variable $x$, we obtain

$$
\partial_{t}\left(\partial_{x} w_{j}\right)=\partial_{x} v \partial_{1} F_{j}+\partial_{x} w_{j} \partial_{2} F_{j}, \quad j=1, \ldots, k,
$$

and

$$
\partial_{t}\left(\partial_{x} z_{i}\right)=\sum_{j=1}^{N} \partial_{x} \varrho_{j} \partial_{j} G_{i}+\partial_{x} v \partial_{N+1} G_{i}+\sum_{j=1}^{k} \partial_{x} w_{j} \partial_{j+N+1} G_{i}+\sum_{j=1}^{m} \partial_{x} z_{j} \partial_{k+N+1+j} G_{i}, \quad i=1, \ldots, m .
$$

Without loss of generality $\partial_{x}$ is the space derivative over one direction (here could be the first, the second or the third dimension of the space $\mathbb{R}^{3}$ ).

According to Lemma 2.1. Lemma 2.2 and the hypothesis (2.5)-2.7), there exist a constant $C$ depending on $T$, such that

$$
\left\|\partial_{1} F_{j}\right\|_{L^{2}(Q)}^{2}+\left\|\partial_{2} F_{j}\right\|_{L^{2}(Q)}^{2} \leqslant C, \quad j=1, \ldots, k
$$


and

$(7.35)$

$\sum_{j=1}^{N}\left\|\partial_{x} \varrho_{j} \partial_{j} G_{i}\right\|_{L^{2}(Q)}^{2}+\left\|\partial_{N+1} G_{i}\right\|_{L^{2}(Q)}^{2}+\sum_{j=1}^{k}\left\|\partial_{j+N+1} G_{i}\right\|_{L^{2}(Q)}^{2}+\sum_{j=1}^{m}\left\|\partial_{k+N+1+j} G_{i}\right\|_{L^{2}(Q)}^{2} \leqslant C, \quad i=1, \ldots, m$.

Multiplying the equation (7.32) by $\left(\partial_{x} w_{j}\right), j=1, \ldots, k$, the equation (7.33) by $\left(\partial_{x} z_{i}\right), i=1, \ldots, m$, and integrating the sum over $\Omega$, and applying the Young inequality of the right hand side, we obtain

$$
\frac{d}{d t}\left(\left\|\partial_{x} \mathbf{w}\right\|_{L^{2}(\Omega)^{k}}^{2}+\left\|\partial_{x} \mathbf{z}\right\|_{L^{2}(\Omega)^{m}}^{2}\right) \leqslant C\left(\left\|\partial_{x} v\right\|_{L^{2}(\Omega)}^{2}+\left\|\partial_{x} \mathbf{w}\right\|_{L^{2}(\Omega)^{k}}^{2}+\left\|\partial_{x} \mathbf{z}\right\|_{L^{2}(\Omega)^{m}}^{2}\right) .
$$

Thanks to the regularity (7.22), we have $\left\|\partial_{x} v\right\|_{L^{2}(\Omega)} \in C^{0}[0, T]$. Applying Gronwall lemma and using hypothesis (2.23), we obtain

$$
\left\|\partial_{x} \mathbf{w}\right\|_{L^{2}(\Omega)^{k}}^{2}+\left\|\partial_{x} \mathbf{z}\right\|_{L^{2}(\Omega)^{m}}^{2} \leqslant C\left(\left\|\partial_{x} \mathbf{w}_{0}\right\|_{L^{2}(\Omega)^{k}},\left\|\partial_{x} \mathbf{z}_{0}\right\|_{\left.L^{2}(\Omega)^{m}\right)}, \quad \forall t \in[0, T] .\right.
$$

Thus,

$$
\mathbf{w} \in L^{\infty}\left(0, T ; H^{1}(\Omega)\right)^{k}, \quad \text { and } \quad \mathbf{z} \in L^{\infty}\left(0, T ; H^{1}(\Omega)\right)^{m} .
$$

On the other hand, from (7.32) and (7.33), we deduce

$$
\mathbf{w} \in W^{1, \infty}\left(0, T ; H^{1}(\Omega)\right)^{k}, \quad \text { and } \quad \mathbf{z} \in W^{1, \infty}\left(0, T ; H^{1}(\Omega)\right)^{m} .
$$

The proof of Proposition 2.1 is finished.

Proof of Proposition 2.2.

Let $p=\partial_{t} v$. We have

$$
\partial_{t} p-\operatorname{div}(\boldsymbol{\sigma} \nabla p)=\partial_{t} I_{a p p}+\partial_{t} I_{i o n}
$$

with initial condition

$$
p(t=0)=\partial_{t} v(t=0)=\operatorname{div}\left(\sigma \nabla v_{0}\right)+I_{\text {app }}(t=0)+I_{\text {ion }}(t=0) .
$$

We start by showing that $p(t=0) \in H^{2}(\Omega)$ in order to be able to apply Proposition 2.1. Using hypotheses 2.9) and $v_{0} \in H^{4}(\Omega)$, we have $\operatorname{div}\left(\sigma \nabla v_{0}\right) \in H^{2}(\Omega)$. We also have $I_{a p p}(t=0) \in H^{2}(\Omega)$. It's obvious that

$$
I_{\text {ion }}(t=0)=\sum_{i=1}^{N} \bar{\varrho}_{i} y_{i}\left(v_{0}\right) \prod_{j=1}^{k} w_{0, j}^{p_{j, i}}\left(v_{0}-E_{i}\left(\mathbf{z}_{0}\right)\right) \in L^{2}(\Omega) .
$$

Thanks to hypothesis (2.12) and the fact the $\bar{\varrho}$ is bounded in $H^{3}(\Omega)^{N}$, there exists a constant $C>0$ such that

$$
\begin{aligned}
\left|\partial_{x} I_{\text {ion }}(t=0)\right|^{2} & \leqslant C\left(\left|\partial_{x} v_{0}\right|^{2}+\left|\partial_{x} \mathbf{w}_{0}\right|^{2}+\left|\partial_{x} \mathbf{z}_{0}\right|^{2}\right), \\
\left|\partial_{x y} I_{i o n}(t=0)\right|^{2} & \leqslant C\left(\left|\partial_{x} v_{0}\right|^{2}+\left|\partial_{x} \mathbf{w}_{0}\right|^{2}+\left|\partial_{x} \mathbf{z}_{0}\right|^{2}+\left|\partial_{y} v_{0}\right|^{2}+\left|\partial_{y} \mathbf{w}_{0}\right|^{2}+\left|\partial_{y} \mathbf{z}_{0}\right|^{2}\right. \\
& \left.+\left|\partial_{x y} v_{0}\right|^{2}+\left|\partial_{x y} \mathbf{w}_{0}\right|^{2}+\left|\partial_{x y} \mathbf{z}_{0}\right|^{2}\right) .
\end{aligned}
$$

So, from the assumption of Proposition 2.2. and $v_{0} \in H^{4}(\Omega), \mathbf{w}_{0} \in H^{2}(\Omega)^{k}, \mathbf{z}_{0} \in H^{2}(\Omega)^{m}$, we deduce that $\partial_{x} I_{i o n}(t=0)$ and $\partial_{x y} I_{i o n}(t=0)$ belong to $L^{2}(\Omega)$. Thus $I_{i o n}(t=0) \in H^{2}(\Omega)$, and then $p(t=0) \in H^{2}(\Omega)$.

On the other hand, we have

$$
v \in H^{1}\left(0, T ; H^{1}(\Omega)\right), \quad \mathbf{w} \in W^{1, \infty}\left(0, T ; H^{1}(\Omega)\right)^{k}, \quad \text { and } \quad \mathbf{z} \in W^{1, \infty}\left(0, T ; H^{1}(\Omega)\right)^{m},
$$

then $I_{i o n} \in H^{1}\left(0, T ; H^{1}(\Omega)\right)$.

Applying Proposition 2.1 to deduce that the solution $p$ verify

$$
p:=\partial_{t} v \in H^{1}\left(0, T ; H^{1}(\Omega)\right) .
$$


Thus

$$
\operatorname{div}(\boldsymbol{\sigma} \nabla v)=p-I_{a p p}-I_{\text {ion }} \in H^{1}\left(0, T ; H^{1}(\Omega)\right)
$$

then

$$
v \in L^{2}\left(0, T ; H^{3}(\Omega)\right)
$$

Also

$$
\operatorname{div}\left(\boldsymbol{\sigma} \nabla \partial_{t} v\right)=\partial_{t} p-\partial_{t} I_{a p p}-\partial_{t} I_{i o n} \in L^{2}\left(0, T ; H^{1}(\Omega)\right)
$$

then

$$
\partial_{t} v \in L^{2}\left(0, T ; H^{3}(\Omega)\right)
$$

Thus

$$
v \in H^{1}\left(0, T ; H^{3}(\Omega)\right) \hookrightarrow \mathcal{C}^{0}\left([0, T] ; \mathcal{C}^{1}(\bar{\Omega})\right) .
$$

Always from Proposition 2.1, we have

$$
\begin{aligned}
\partial_{t} v:=p \in L^{2}\left(0, T ; H^{2}(\Omega)\right) & \Rightarrow \quad v \in H^{1}\left(0, T ; H^{2}(\Omega)\right), \\
\partial_{t} v:=p \in L^{\infty}\left(0, T ; H^{2}(\Omega)\right) & \Rightarrow \quad v \in W^{1, \infty}\left(0, T ; H^{2}(\Omega)\right), \\
\partial_{t} v:=p \in H^{2}\left(0, T ; L^{2}(\Omega)\right) & \Rightarrow \quad v \in H^{3}\left(0, T ; L^{2}(\Omega)\right) .
\end{aligned}
$$

Let us now prove the regularities (2.26). Getting second derivatives of equations (2.3) and (2.5) over the space variable $x$, multiplying both equations by $\partial_{x x} w_{j}$ and $\partial_{x x} z_{i}$ respectively and using that fact that $\bar{\varrho}$ is bounded in $H^{3}(\Omega)^{N}$, integrating the sum over $\Omega$ and applying the Young inequality of the right hand side, we obtain

$$
\frac{d}{d t}\left(\left\|\partial_{x x} \mathbf{w}\right\|_{L^{2}(\Omega)^{k}}^{2}+\left\|\partial_{x x} \mathbf{z}\right\|_{L^{2}(\Omega)^{m}}^{2}\right) \leqslant C_{1}\left(\left\|\partial_{x x} \mathbf{w}\right\|_{L^{2}(\Omega)^{k}}^{2}+\left\|\partial_{x x} \mathbf{z}\right\|_{L^{2}(\Omega)^{m}}^{2}\right)+C_{2},
$$

where $C_{1}$ and $C_{2}$ are two non negative constants.

From [2.23), 7.51] and applying Gronwall Lemma, we obtain

$$
\left\|\partial_{x x} \mathbf{w}\right\|_{L^{2}(\Omega)^{k}}^{2}+\left\|\partial_{x x} \mathbf{z}\right\|_{L^{2}(\Omega)^{m}}^{2} \leqslant C\left(\left\|\partial_{x x} \mathbf{w}_{0}\right\|_{L^{2}(\Omega)^{k}},\left\|\partial_{x x} \mathbf{z}_{0}\right\|_{L^{2}(\Omega)^{m}}\right) .
$$

Thus,

$$
\mathbf{w} \in L^{\infty}\left(0, T ; H^{2}(\Omega)\right)^{k}, \quad \text { and } \quad \mathbf{z} \in L^{\infty}\left(0, T ; H^{2}(\Omega)\right)^{m} .
$$

Similarly, computing the third space derivatives of (2.3) and (2.5), multiplying both equations by $\partial_{x x x} w_{j}$ and $\partial_{x x x} z_{i}$ respectively and using that fact that $\varrho$ is bounded in $H^{3}(\Omega)^{N}$, integrating the sum over $\Omega$ and applying the Young inequality of the right hand side, we obtain

$$
\frac{d}{d t}\left(\left\|\partial_{x x x} \mathbf{w}\right\|_{L^{2}(\Omega)^{k}}^{2}+\left\|\partial_{x x x} \mathbf{z}\right\|_{L^{2}(\Omega)^{m}}^{2}\right) \leqslant C_{3}\left(\left\|\partial_{x x x} \mathbf{w}\right\|_{L^{2}(\Omega)^{k}}^{2}+\left\|\partial_{x x x} \mathbf{z}\right\|_{L^{2}(\Omega)^{m}}^{2}\right)+C_{4} .
$$

since $v \in L^{\infty}\left(0, T ; H^{3}(\Omega)\right)$, where $C_{3}$ and $C_{4}$ are two non negative constants. Using $\mathbf{w}_{0} \in H^{3}(\Omega)^{k}$, $\mathbf{z}_{0} \in H^{3}(\Omega)^{m}$ and applying Gronwall Lemma, we have

$$
\left\|\partial_{x x x} \mathbf{w}\right\|_{L^{2}(\Omega)^{k}}^{2}+\left\|\partial_{x x x} \mathbf{z}\right\|_{L^{2}(\Omega)^{m}}^{2} \leqslant C\left(\left\|\partial_{x x x} \mathbf{w}_{0}\right\|_{L^{2}(\Omega)^{k}},\left\|\partial_{x x x} \mathbf{z}_{0}\right\|_{L^{2}(\Omega)^{m}}\right) .
$$

Then

$$
\mathbf{w} \in L^{\infty}\left(0, T ; H^{3}(\Omega)\right)^{k}, \quad \text { and } \quad \mathbf{z} \in L^{\infty}\left(0, T ; H^{3}(\Omega)\right)^{m} .
$$

Moreover, from the expressions of $\partial_{t}\left(\partial_{x x x} \mathbf{w}\right)$ and $\partial_{t}\left(\partial_{x x x} \mathbf{z}\right)$, we deduce 


$$
\begin{aligned}
\mathbf{w} \in W^{1, \infty}\left(0, T ; H^{3}(\Omega)\right)^{k} \subset H^{1}\left(0, T ; H^{3}(\Omega)\right)^{k} & \hookrightarrow \mathcal{C}^{0}\left([0, T] ; \mathcal{C}^{1}(\bar{\Omega})\right)^{k} \\
\mathbf{z} \in W^{1, \infty}\left(0, T ; H^{3}(\Omega)\right)^{m} \subset H^{1}\left(0, T ; H^{3}(\Omega)\right)^{m} & \hookrightarrow \mathcal{C}^{0}\left([0, T] ; \mathcal{C}^{1}(\bar{\Omega})\right)^{m}
\end{aligned}
$$

Acknowledgements. This work has been supported by EPICARD cooperative research program, funded by INRIA international laboratory LIRIMA. The LAMSIN researcher's work is supported on a regular basis by the Tunisian Ministry of Higher Education, Scientific Research and Technology.

\section{REFERENCES}

[1] R. A. Adams. Sobolev Spaces. Academic Press, New Tork, 1975.

[2] G. W. Beeler and H. Reuter. Reconstruction of the action potential of ventricular myocardial fibres. The Journal of physiology, 268(1):177, 1977.

[3] M. Bellassoued. Global logarithmic stability in inverse hyperbolic problem by arbitrary boundary observation. Inverse Problems, 20(4):1033-1052, 2004.

[4] M. Bellassoued and M. Yamamoto. Logarithmic stability in determination of a coefficient in an acoustic equation by arbitrary boundary observation. J. Math. Pures Appl. (9), 85(2):193-224, 2006.

[5] M. Boulakia and E. Schenone. Stability estimates for some parameters of a reaction-diffusion equation coupled with an ode. Applicable Analysis, 96(7):1138-1145, 2017.

[6] M. Boulakia, E. Schenone, and J-F. Gerbeau. Reduced-order modeling for cardiac electrophysiology. application to parameter identification. International Journal for Numerical Methods in Biomedical Engineering, 28(6-7):727-744, 2012.

[7] A. L. V. Bukhgeim and M. V. Klibanov. Uniqueness in the magnitude of one class of miltidimensional inverse problems. Doklady Akademii Nauk SSSR, 260(2):269-272, 1981.

[8] D. Chapelle, A. Gariah, P. Moireau, and J. Sainte-Marie. A galerkin strategy with proper orthogonal decomposition for parameter-dependent problems-analysis, assessments and applications to parameter estimation. ESAIM: Mathematical Modelling and Numerical Analysis, 47(6):1821-1843, 2013.

[9] J. Cheng and M. Yamamoto. One new strategy for a priori choice of regularizing parameters in Tikhonov's regularization. Inverse Problems, 16(4):L31-L38, 2000.

[10] A. Collin, D. Chapelle, and P. Moireau. A luenberger observer for reaction-diffusion models with front position data. Journal of Computational Physics, 300:288-307, 2015.

[11] C. Corrado, J-F. Gerbeau, and P. Moireau. Identification of weakly coupled multiphysics problems. application to the inverse problem of electrocardiography. Journal of Computational Physics, 283:271-298, 2015.

[12] A. Elayyan and V. Isakov. On uniqueness of recovery of the discontinuous conductivity coefficient of a parabolic equation. SIAM J. Math. Anal., 28(1):49-59, 1997.

[13] L. P. Endresen, K. Hall, J. S. Hoye, and J. Myrheim. A theory for the membrane potential of living cells. European Biophysics Journal, 29(2):90-103, 2000.

[14] R. FitzHugh. Impulses and physiological states in theoretical models of nerve membrane. Biophysical journal, 1(6):445, 1961.

[15] P. C. Franzone and L. F. Pavarino. A parallel solver for reaction-diffusion systems in computational electrocardiology. Mathematical Models and Methods in Applied Sciences, 14(06):883-911, 2004.

[16] P. C. Franzone, L. F. Pavarino, and S. Scacchi. Mathematical cardiac electrophysiology, volume 13. Springer, 2014.

[17] A. V. Fursikov and O. Yu. Imanuvilov. Controllability of evolution equations. Seoul National University, RIM, Seoul, South Korea, 34, 1996.

[18] A. V. Fursikov and O. Yu. Imanuvilov. Exact controllability of the navier-stokes and boussines equations. Russian Mathematical Surveys, 54(3):565-618, 1999.

[19] A. L. Hodgkin and A. F. Huxly. A quantitative description of membrane current and its application to conduction and excitation in nerve. The Journal of Physiology, 117(4):500-544, 1952.

[20] O. Yu. Imanuvilov and M. Yamamoto. Lipschitz stability in inverse parabolic problems by the Carleman estimate. Inverse Problems, 14(5):1229-1245, 1998.

[21] O. Yu. Imanuvilov and M. Yamamoto. Carleman estimate for a parabolic equation in a Sobolev space of negative order and its applications. In Control of nonlinear distributed parameter systems (College Station, TX, 1999), volume 218 of Lecture Notes in Pure and Appl. Math., pages 113-137. Dekker, New York, 2001.

[22] O. Yu. Imanuvilov and M. Yamamoto. Determination of a coefficient in an acoustic equation with a single measurement. Inverse Problems, 19(1):157-171, 2003. 
[23] V. Isakov. Inverse problems for partial differential equations, volume 127 of Applied Mathematical Sciences. Springer-Verlag, New York, 1998.

[24] V. Isakov and S. Kindermann. Identification of the diffusion coefficient in a one-dimensional parabolic equation. Inverse Problems, 16(3):665-680, 2000.

[25] M. Ivanchov. Inverse problems for equations of parabolic type, volume 10 of Mathematical Studies Monograph Series. VNTL Publishers, L/viv, 2003.

[26] T. Kato. Perturbation theory for linear operators. Die Grundlehren der mathematischen Wissenschaften, 132:Springer-Verlag New York, Inc., New York, 1980.

[27] A. Khaidarov. Carleman estimates and inverse problems for second-order hyperbolic equations. Mat. Sb. (N.S.), 130(172)(2):265-274, 287, 1986.

[28] M. V. Klibanov. Inverse problems in the "large" and Carleman estimates. Differentsialınye Uravneniya, 20(6):1035-1041, 1984.

[29] M. V. Klibanov. Inverse problems and Carleman estimates. Inverse Problems, 8(4):575-596, 1992.

[30] M. V. Klibanov and A. Timonov. Carleman estimates for coefficient inverse problems and numerical applications. Inverse and Ill-posed Problems Series. VSP, Utrecht, 2004.

[31] M. V. Klibanov and M. Yamamoto. Lipschitz stability of an inverse problem for an acoustic equation. Appl. Anal., 85(5):515538, 2006.

[32] E. Konukoglu, J. Relan, U. Cilingir, B. Menze, P. Chinchapatnam, A. Jadidi, H. Cochet, M. Hocini, H. Delingette, P. Jaïs, M. Haïssaguerre, N. Ayache, and M. Sermesant. Efficient probabilistic model personalization integrating uncertainty on data and parameters: Application to eikonal-diffusion models in cardiac electrophysiology. Progress in Biophysics and Molecular Biology, 107(1):134-146, Oct 2011.

[33] J. L. Lions and E. Magnenes. Non-homogeneous Boundary Value Problems and Applications. Springer-Verlag, Berlin, 1972.

[34] C. H. Luo and Y. Rudy. A model of the ventricular cardiac action potential: Depolarization, repolarization, and their interaction. Circulation Research, 68(6):1501-1526, 1991.

[35] C. H. Luo and Y. Rudy. A dynamic model of the cardiac ventricular action potential. i. simulations of ionic currents and concentration changes. Circulation Research, 74(6):1071-1096, 1994.

[36] M. Mahjoub, J. Lassoued, and N. Zemzemi. Stability results for the parameter identification inverse problem in cardiac electrophysiology. Inverse Problems, 32(11), 2016.

[37] S. Marchesseau, H. Delingette, M. Sermesant, and N. Ayache. Fast parameter calibration of a cardiac electromechanical model from medical images based on the unscented transform. Biomechanics and modeling in mechanobiology, pages 1-17, 2013.

[38] C. C. Mitchell and D. G. Schaeffer. A two-current model for the dynamics of cardiac membrane. Bulletin of mathematical biology, 65(5):767-793, 2003.

[39] J.C. Neu and W. Krassowska. Homogenization of syncytial tissues a comparison of monodomain and bidomain reactiondiffusion models for action potential propagation in the human heart. IEEE Transactions on Biomedical Engineering, 21(2), 1993.

[40] D. Ngoma, P. Vianney, Y. Bourgault, and H. Nkounkou. Parameter identification for a non-differentiable ionic model used in cardiac electrophysiology. Applied Mathematical Sciences, 9(150):7483-7507, 2015.

[41] M. Veneroni. Reaction-diffusion systems for the microscopic cellular model of the cardiac electric field. Mathematical methods in the applied sciences, 29(14):1631-1661, 2006.

[42] M. Veneroni. Reaction-diffusion systems for the macroscopic bidomain model of the cardiac electric field. Nonlinear Analysis: Real World Applications, 10:849-868, 2009.

[43] M. Yamamoto. Uniqueness and stability in multidimensional hyperbolic inverse problems. J. Math. Pures Appl. (9), 78(1):6598, 1999.

[44] M. Yamamoto and J. Zou. Simultaneous reconstruction of the initial temperature and heat radiative coefficient. Inverse Problems, 17(4):1181-1202, 2001. Special issue to celebrate Pierre Sabatier's 65th birthday (Montpellier, 2000).

[45] G. Yuan and M. Yamamoto. Lipschitz stability in the determination of the principal part of a parabolic equation. ESAIM: COCV, 15(3):525-554, 2009.

${ }^{1}$ Tunis El Manar University, ENIT-LAMSin, BP 37, Le Belvédère 1002 Tunis, Tunisia

E-mail address: yassine.abidi@enit.rnu.tn

E-mail address: mourad.bellassoued@enit.utm.tn

E-mail address: moncef.mahjoub@lamsin.rnu.tn

2 INRIA, Bordeaux Sud-Ouest, 200 Avenue de la Vielle Tour 33405 TAlence Cedex France

E-mail address: nejib.zemzemi@inria.fr 